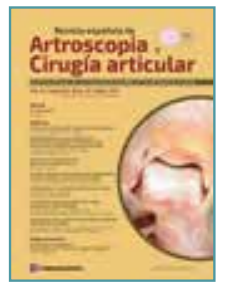

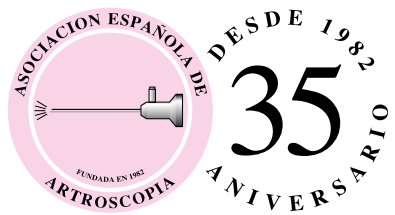

Vol. 24. Especial. Núm. 57. Mayo 2017

ISSN: 2386-3129 (impreso)

2443-9754 (online)

\title{
Original
}

\section{Historia de la reparación del ligamento cruzado anterior}

\author{
M. Leyes ${ }^{1}$, F. Forriol ${ }^{2}$ \\ ${ }^{7}$ Equipo Leyes, Flores y Asociados. Clínica CEMTRO. Madrid \\ ${ }^{2}$ Facultad de Medicina. Universidad San Pablo CEU. Campus de Montepríncipe. Boadilla del Monte, Madrid
}

Correspondencia:

Recibido el 10 de marzo de 2017

Dr. Manuel Leyes Vence

Aceptado el 22 de marzo de 2017

Correo electrónico: leyesm@yahoo.com

Disponible en Internet: mayo 2017

\section{RESUMEN}

La reconstrucción del ligamento cruzado anterior es hoy una de las cirugías más frecuentes en el mundo. Sin embargo, ha supuesto un reto para los cirujanos desde principios del siglo XX y todavía quedan aspectos técnicos y biomecánicos controvertidos. La artroscopia ha facilitado mucho la recuperación de los pacientes y los resultados de larga evolución. Muchos autores han participado en su desarrollo, aunque algunos han quedado en el olvido.

Conocer los pasos y las opciones de las diferentes escuelas nos puede ayudar a comprender mejor lo que se hace hoy, preparar nuevos retos para el futuro y saber que se sigue escribiendo la historia.

Palabras clave: Ligamento cruzado anterior. Plastia. Injerto. Inestabilidad. Rodilla.

\section{Las roturas del ligamento cruzado anterior: los orígenes}

Los ligamentos cruzados de la rodilla son los encargados de regular la cinemática articular y son

\begin{abstract}
History of anterior cruciate ligament repair

The reconstruction techniques of the anterior cruciate ligament are one of the most frequent surgeries in the world. However, it has been a challenge for surgeons since the beginning of the $20^{\text {th }}$ century and there are still controversial technical and biomechanical aspects. Arthroscopy has greatly facilitated patient recovery and long-term outcomes. Many authors have participated in its development, although some have been forgotten.

Knowing the steps and the options of the different schools can help us to better understand what is being done today, to prepare new challenges for the future and to know that the history continues to be written.
\end{abstract}

Key words: Anterior cruciate ligament. Graft. Plasty. Instability. Knee.

también los "órganos sensores" que informan de la musculatura periarticular, influyendo sobre la posición de las superficies articulares, la dirección y la magnitud de las fuerzas y, de forma indirecta, distribuyen las tensiones articulares ${ }^{(1)}$. Con

https://doi.org/10.24129/j.reaca.24e57.fs1703010

FS $\odot 2017$ Fundación Española de Artroscopia. Publicado por Imaidea Interactiva en FONDOSCIENCE ${ }^{\circledR}$ (www.fondoscience.com). Este es un artículo Open Access bajo la licencia CC BY-NC-ND (www.creativecommons.org/licenses/by-nc-nd/4.0/). 


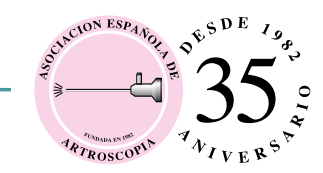

una visión clínica, la primera función del ligamento cruzado anterior (LCA) es impedir el desplazamiento anterior de la tibia con relación al fémur $y$, secundariamente, prevenir la laxitud en varo o en valgo y la rotación ${ }^{(2,3)}$.

Reflejamos los aspectos de mayor interés en esta larga historia, donde se entremezclan aspectos técnicos y se cruzan ideas; además, en ocasiones, aparecen modas que son pasajeras y quedan en el olvido. La cirugía del LCA tiene unos orígenes claros, cumple ahora casi cien años, si bien como veremos algunos cirujanos fueron visionarios y atrevidos y llegaron mucho antes de tiempo. Nos vamos a basar en trabajos previos ${ }^{(4-7)}$ para seguir comprendiendo la evolución de esta cirugía.

La anatomía y la biomecánica de la rodilla y de los ligamentos cruzados comenzó con los hermanos Weber -Wilhelm, profesor de física en Gotinga, y Eduard, profesor de anatomía y fisiología en Leipzig-, describiendo la posición anatómica de los dos ligamentos cruzados y mostrando cómo sus fascículos se tensaban, según el grado de flexión, de manera diferente ${ }^{(8)}$; Karl Langer ${ }^{(9)}$, anatómico vienés, presentó la cinemática de la articulación y la función de los ligamentos cruzados en la rotación. Los trabajos experimentales realizados sobre cadáveres de Dittel en Viena ${ }^{(10)}$, Bonnet en Lyon $^{(11)}$ y Hönigschmied ${ }^{(12)}$ demostraron que el LCA rompía, en la mayoría de las ocasiones, cerca de su inserción femoral, aunque no era raro ver, como señaló Paul Segond ${ }^{(13)}$ en París, un arrancamiento óseo de la tibia dejando el ligamento intacto.

En la segunda mitad del siglo XIX, anatómicos y fisiólogos, como Hermann von Meyer ${ }^{(14)}$, Hermann Zuppinger ${ }^{(15)}$ y Hans Straßer ${ }^{(16)}$, explicaron el funcionamiento del LCA y Robert Fick ${ }^{(17)}$, en Leipzig, describió el modelo de 4 barras para explicar la movilidad articular, señalando con detalle la tensión a la que se ven sometidas las diferentes fibras del LCA y mencionando, de pasada, el concepto de la isometría. Bruno Pfab ${ }^{(18)}$, en Graz, describió, por su parte, la vascularización del LCA. No sería hasta bien entrado el S. XX cuando, en 1941 Otto Brantigan y Allan Vorshell(19), ambos en Baltimore, y LeRoy Abbott ${ }^{(20)}$, en San Francisco en 1944, comenzaron a ver el LCA como una estructura básica en la biomecánica de la articulación de la rodilla. Consideraron al LCA el primer estabilizador en la traslación anterior de la tibia sobre el fémur y un estabilizador secundario de la rotación. Estos trabajos continuarian con las publicaciones de Fakhry
Girgis $^{(21)}$, de Nueva York, en 1975, y Lyle Norwood ${ }^{(22)}$, de Columbus, en 1979, entre otros.

Durante muchos años, las roturas del LCA se encontraron con la falta de un diagnóstico certero y seguro; muchas lesiones pasaban desapercibidas o mostraban una evidente inestabilidad tiempo después de producirse, lo que complicaba todavía más la patología. En 1875, el griego Georgios C. Noulis ${ }^{(7)}$ describió una maniobra muy semejante a la prueba de Lachman, publicada mucho más tarde, en 1976, por Joseph Torg ${ }^{(23)}$ y sus colaboradores en Filadelfia, a la que este cedió el epónimo de su maestro John Lachman (24). Además, era difícil determinar y clasificar el tipo de lesión y tampoco se contaba con las técnicas adecuadas para reparar un LCA roto.

Hoy vemos con la mayor naturalidad la cirugía del LCA, una de la más frecuentes, y, sin embargo, no hace tanto, en 1974, Kennedy ${ }^{(25)}$ escribía en la introducción de uno de sus artículos que "una atmósfera de incertidumbre invade el quirófano cuando se descubre una lesión del LCA al efectuar una artrotomía. Entre las autoridades de la rodilla hay desacuerdo sobre si se puede producir una lesión aislada del LCA y cuándo se produce y cómo afecta a la función de la articulación". La dificultad de reparación del LCA se explica por motivos biológicos y anatómicos. Es un ligamento cordonal rodeado por sinovial al que le resulta difícil cicatrizar y recuperar sus condiciones biomecánicas. Palmer (26), en 1938, estableció que "una rotura total de un fascículo del ligamento cruzado anterior es incapaz de curar espontáneamente", lo cual se debe a la falta de vasos y a la temprana atrofia del ligamento. Arnoczky et al. ${ }^{(27,28)}$ demostraron que la vascularización del LCA era de proximal a distal. Por el contrario, el aporte vascular del ligamento cruzado posterior (LCP) es de distal a proximal(29), lo cual explica que al romperse el LCA en su inserción proximal se atrofia de inmediato al desaparecer su aporte vascular.

\section{La sutura directa de un ligamento cruzado anterior roto}

En el siglo XIX se publicaron trabajos relacionados con la clínica y el diagnóstico de las roturas del LCA. Una de las primeras fue la experiencia de Robert Adams(30), quien tuvo la oportunidad de hacer la autopsia a un joven quien, ebrio y 
como consecuencia de una pelea, murió a los pocos días. La rodilla presentaba una infección y un arrancamiento tibial del LCA. Al mismo tiempo, Amedée Bonnet ${ }^{(11)}$, profesor de cirugía de Lyon, estableció la clínica de la rotura del LCA. Recomendaba el tratamiento conservador y una movilización temprana.

Los primeros cirujanos propugnaron el sistema más sencillo y lógico, suturar lo antes posible los cabos del LCA roto. Fue James Stark ${ }^{(31)}$, en 1850, en Edimburgo, quien presentó los primeros resultados en 2 pacientes con rotura del LCA a los que realizó un tratamiento conservador, con 3 meses de inmovilización y 10 meses con férula semirrígida. Después, la primera reparación de una lesión aguda de LCA fue efectuada, en 1903, por Mayo Robson(32), en el General Infirmary, de Leeds. En 1907, Fritz Lange ${ }^{(33)}$ suturó con seda un LCA; en 1910, Martin Kirschner(34), de Königsberg, y en 1913 John Davis(35), de Baltimore, publicaron su experiencia. Se conocen también las cirugías efectuadas por Edgar Bick(36), en Nueva York, Richard Warren y William Blake, en Londres. En 1913, Herbert Goetjes ${ }^{(37)}$, de Colonia, presentó sus resultados en 23 pacientes con el LCA suturado sin apreciar una movilidad anormal y recomendaba la exploración de la rodilla bajo anestesia cuando existían dudas en su diagnóstico. También Georg Perthes ${ }^{(38)}$, en Tubinga, viendo la dificultad de reconstruir un LCA roto y de hacer una sutura directa, propuso unir la porción del ligamento roto al hueso con un alambre de bronce y aluminio, pasando los cabos del alambre por 2 perforaciones femorales que anudaba en el exterior, además de suturar el LCA. De hecho, cuando Ivar Palmer ${ }^{(26)}$ publicó su tesis doctoral en 1938 todavía recomendaba la técnica de Perthes en las fases muy tempranas de la rotura.

La experiencia clínica pronto avaló la ineficacia de las suturas y la única diferencia apreciable respecto al tratamiento conservador era una menor incidencia de signos de inestabilidad ${ }^{(39)}$. Jonash ${ }^{(40)}$ vio que las lesiones tratadas con medios conservadores tenían un 25\% de malos resultados, un $50 \%$ de regulares y solo un $25 \%$ de buenos. Todavía O'Donoghue ${ }^{(41)}$, en 1955, señalaba que la cirugía solo estaba indicada cuando los síntomas de la insuficiencia del LCA eran tan graves que interferían en las actividades diarias. Pero, por aquellos años, cirujanos de reconocido prestigio ${ }^{(42,43)}$ sostenían que el LCA no necesitaba ninguna reparación si el cartílago y los meniscos estaban intactos.
La sutura del LCA, con sus diferentes modificaciones, se siguió utilizando hasta los años ochenta con buenos resultados, con variaciones de la técnica de Perthes y con suturas que pasaban por detrás del cóndilo lateral del fémur, over-thetop ${ }^{(43,44)}$. La experiencia recogida llevó a Frank Noyes ${ }^{(44)}$, en 1983, a establecer la regla de los tercios; un tercio de los pacientes compensan su lesión y hacen una vida completamente normal, otro tercio hacen vida normal abandonando su actividad deportiva o laboral y el último tercio lleva una vida con problemas.

Históricamente, se han desarrollado 2 sistemas de reconstrucción del LCA, las técnicas intraarticulares y las técnicas extraarticulares. En ocasiones, se ha utilizado una combinación de ambas. La dificultad inicial de reconstruir el LCA obligó a buscar soluciones para tratar la inestabilidad sin necesidad de abrir la articulación. Las técnicas intraarticulares sustituyen el LCA lesionado, mientras que las técnicas extraarticulares lo hacen a distancia de la inserción del propio LCA. No siempre son fáciles de distinguir y en su desarrollo han aparecido de manera simultánea.

\section{La reconstrucción del ligamento cruzado anterior}

Erwin Payr ${ }^{(45)}$, en Leipzig, conociendo que la porción proximal del LCA roto suele ser corta, realizó la primera reconstrucción del ligamento con una lazada que obtenía de una tira de fascia lata. En 1913, un cirujano italiano, Nicoletti, fue el primero en realizar experimentalmente una reconstrucción de un LCA en un perro utilizando una plastia con un injerto libre ${ }^{(4)}$. Paul Wagner ${ }^{(46)}$ leyó su tesis doctoral en 1913, en Leipzig, sobre la reconstrucción con fascia de LCA rotos. En 1913, Knut Giertz ${ }^{(47)}$ operó a una niña de 13 años con una artritis séptica potenciando los ligamentos colaterales y seccionando la fascia lata para mejorar la estabilidad.

Más interesante fue la aportación de Iván Grekov, en San Petersburgo, quien, estimulado por su ayudante Erich Hesse ${ }^{(48)}$, realizó la primera reconstrucción conocida de un LCA con injerto libre de fascia fijado, por medio de perforaciones, al fémur y, por el otro lado, suturado al remanente distal del LCA ${ }^{(6)}$.

En 1918, Hermann Matti(49), de Berna, utilizó el injerto de fascia lata desde la cara posterior del 


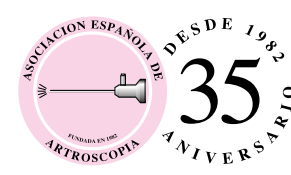

cóndilo interno del fémur oblicuamente hasta la cara anterointerna de la tibia y en las inestabilidades del LCP invertía el recorrido. Granville Bennett ${ }^{(50)}$, en Boston, siguió la técnica de Matti para reforzar el ligamento lateral interno (LLI). Colocaba una tira de fascia lata en la cara interna de la rodilla asociada a un tensado del retináculo extensor medial, pensando que un LCA roto se puede suplir si el resto de los ligamentos articulares están intactos. Los resultados fueron buenos y uno de sus pacientes jugó como capitán del equipo de fútbol americano, los All American, un año después de la cirugía. En 1923, Bertocchi y Bianchetti(51) estudiaron las propiedades mecánicas y la evolución histológica de los autoinjertos de fascia lata y del tendón de Aquiles del cerdo. Es el primer estudio experimental de la relación de un sustitutivo ligamentoso. Zanoli(52), en 1926, tras extraer un trozo de la cintilla iliotibial, la mantuvo durante más de 1 mes subcutánea en el tejido graso del paciente, para implantarla, posteriormente, sustituyendo al LCA.

Un momento importante en la historia de la cirugía del LCA fue la aportación del irlandés Hey-Groves, en $1917^{(53)}$ y en $1920^{(54)}$, quien desarrolló, en Bristol, la primera técnica de reconstrucción del LCA con la cintilla iliotibial. Desinsertaba la fascia lata de la tibia, con sus vasos y nervios, y la pasaba por un túnel en el fémur que seguía con otro túnel oblicuo en la tibia. El éxito dependía de la situación anatómica de la plastia. Había que evitar un injerto demasiado vertical. Fue el primero en describir la subluxación anterolateral de la tibia en una rodilla con un LCA deficiente (pivot-shift), como había señalado Bonnet ${ }^{(11)}$ setenta años antes. Tras ver los resultados, modificó su técnica y en 1920 ${ }^{(54)}$ presentó 14 casos de los cuales 4 no habían mejorado. En 1918, Alwyn Smith ${ }^{(55)}$, extrayendo una porción rectangular distal de la cintilla iliotibial, trataba tanto el LLI como el LCA y, como ocurrió con Hey-Groves ${ }^{(53)}$, obtuvo buenos resultados, aunque debemos resaltar su protocolo de rehabilitación y estimulación eléctrica postoperatoria; además, describió la reparación del LCA con múltiples suturas de seda fijadas con grapas metálicas, pero debido a la fuerte sinovitis que producían fueron pronto desechadas. Cubbins et al. ${ }^{(56)}$, en 1932, tras diversos estudios anatómicos y mecánicos, llegaron a la conclusión de que la técnica propuesta por Smith ${ }^{(55)}$ utilizando la aponeurosis del músculo bíceps femoral era óptima.
Inmovilizaban con un yeso a $30^{\circ}$ de flexión durante 30 días y después colocaban otro yeso en extensión completa durante otros 3 meses.

Putti(57), en 1920, preconizó la utilización de la fascia lata y poco después recomendó la utilización del tendón del músculo semimembranoso, mientras que Holzel(58), también en 1920, para reemplazar el ligamento dañado, introducía la parte libre de un menisco roto en asa de cubo. En estos tratamientos siempre había que inmovilizar con un yeso y una férula de movilidad controlada durante un tiempo largo de recuperación. Más tarde, Charles F. Eikenbary ${ }^{(59)}$, en Seattle, mejoró la técnica de Hey-Groves y Putti, utilizando el injerto libre.

En 1927, Alexander Edwards ${ }^{(60)}$, en Glasgow, realizó una reconstrucción del LCA con los tendones de la pata de ganso en el cadáver. En 1934, Ricardo Galeazzi(61), en Milán, fue el primero en utilizar tendón del músculo semitendinoso que dejaba anclado distalmente a la pata de ganso con una técnica muy parecida a la propuesta por Hey-Groves $^{(54)}$. Sus publicaciones fueron en revistas italianas, por lo que no tuvo difusión. De hecho, Harry Macey ${ }^{(62)}$, desconociendo los trabajos de Galeazzi, realizó en la Clínica Mayo de Rochester lo mismo simplificando la técnica con 2 incisiones, técnica que sería modificada en 1974 por James McMaster ${ }^{(63)}$, en Pittsburgh, que utilizaba el músculo recto interno fijado al cóndilo lateral con una grapa. En 1957, Merle D’Aubigné(64), en París, junto con Max Lange ${ }^{(65,66)}$, en Múnich, fueron los primeros en analizar los resultados con plastia de tendón semitendinoso de larga evolución.

El primero en reconstruir el LCA con el ligamento rotuliano fue Mitchell Langworthy ${ }^{(7)}$, de Spokane, Washington, en 1929, en un paciente que no llegó a publicar pues murió ese mismo año por el disparo de un paciente en su consulta. En 1928, Ernst Gold ${ }^{(67)}$, de Viena, utilizó el retináculo y la porción interna del tendón rotuliano que pasó por un túnel tibial y lo suturó a la parte posterosuperior del LCP en una esquiadora que se había roto el LCA 2 años antes. Max zur Verth ${ }^{(68)}$ en las roturas crónicas del LCA, reconstruía con una porción pediculada del tendón rotuliano y aunque nunca presentó su técnica, Arnold Wittek ${ }^{(68)}$, de Graz, la dio a conocer en 16 pacientes con éxito, respetando la autoría de Zur Verth.

Willis C. Campbell(69,70), de Memphis, en 1936, describió su técnica intraarticular, mediante túneles en la tibia y el fémur, empleando el bor- 
de interno del tendón cuadricipital y ligamento rotuliano, efectuando un túnel femoral y tibial como había recomendado Hey-Groves y suturando el injerto al periostio del fémur distal. Destacó, como había hecho antes Dittel ${ }^{(10)}$, la frecuencia de las lesiones combinadas de menisco interno y LLI en las rodillas con rotura del LCA, que denominó la "tríada terrible" y que, años más tarde, O'Donoghue ${ }^{(41,71)}$ consideró la "tríada desgraciada". Ese mismo año, Mauck ${ }^{(72)}$ recomendaba el avance distal de la inserción del LLI, lo cual exigía la resección del menisco interno, para estabilizar una rodilla con el LCA roto. Sin embargo, lo más interesante de su técnica fue la colocación de una ortesis móvil, durante 6-8 semanas.

Cotton y Morrison(73), así como, en 1936, los hermanos David y Bordman Bosworth ${ }^{(74)}$, en Nueva York, seguían manteniendo la idea de que la reconstrucción del LLI, con tiras de fascia lata, transformaba una rodilla deficiente por la rotura del LCA en una rodilla estable, aunque Kennedy y Fowler ${ }^{(75)}$ demostraron que el LCA puede estar lesionado sin que se afecten las estructuras capsulares internas. De hecho, en España, Moragas et al. ${ }^{(76)}$, en 1956, siguiendo las recomendaciones de las publicaciones internacionales, intervenían las rodillas traumáticas de los futbolistas; aunque resulta llamativo que, en su revisión de 26 casos, 21 correspondían a roturas del LLI y tan solo 4 a roturas del LCA.

El citado trabajo de Palmer ${ }^{(26)}$, publicado en 1938, fue la base de los conocimientos básicos de las lesiones ligamentosas de la rodilla, pues describió su anatomía, biomecánica y la indicación del tratamiento. Discutió, por primera vez, el "signo del cajón" y dio cuenta de los hallazgos radiográficos e histológicos de sus cirugías. Palmer ${ }^{(26)}$ utilizó la técnica de Hey-Groves ${ }^{(54)}$ y mencionó la necesidad de efectuar los orificios de los túneles adecuadamente, para lo que desarrolló una guía.

Hauser $^{(77)}$, en 1947, presentó una nueva reconstrucción extraarticular utilizando una porción distal del ligamento rotuliano; lo fijaba, mediante grapas o agujas, en el punto de inserción femoral del LLI, pensando que asi duplicaba la función del LCA y reforzaba las estructuras mediales, tan frecuentemente debilitadas. Por su parte, el mismo año, Arthur Jacob Helfet ${ }^{(78)}$, en Ciudad del Cabo, Sudáfrica, para reforzar el complejo interno de la rodilla, desplazaba la tuberosidad tibial medial, controlando así la rotación externa de la tibia. Además, pasaba el tendón del músculo semitendinoso por un canal longitudinal medial, en línea con el LLI, para provocar la rotación interna del fémur con la flexión. Los resultados eran buenos cuando el paciente era capaz de contraer los isquiotibiales y permanecía la inestabilidad pasiva.

Kurt Lindemann ${ }^{(79)}$, en 1950, propuso la reconstrucción dinámica para reducir la inestabilidad por el efecto estabilizador de la unidad musculotendinosa del músculo recto interno. En realidad, equivalía a una trasposición tendinosa. La plastia de LCA, tomada del músculo recto interno desinsertado, la pasaba por la escotadura para introducirla, a través de un túnel tibial, siendo suturada a su salida con el ligamento rotuliano. Posteriormente, inmovilizaba con un yeso pelvipédico durante 3 semanas y no permitía la carga hasta pasadas 5 o 6 semanas de la cirugía, siempre que la rodilla flexionara activamente de $30^{\circ}$ a $40^{\circ}$. A las 8 semanas, la flexión y la extensión debían ser completas o, al menos, llegar hasta los $90^{\circ(80)}$. Por su parte, Ficat ${ }^{(81,82)}$ sistematizó esta técnica conservando el fundamento "dinámico" de la estabilización articular con la contracción muscular reflejas ante las tensiones ejercidas por el tendón, fijando el tendón sobre el LLI. Bousquet ${ }^{(83)}$ añadió una función "estática", al reintroducir distalmente el tendón en la articulación fijándolo, tras realizar un túnel transóseo, en el cóndilo externo ${ }^{(83-85)}$. En la línea de las reconstrucciones "dinámicas", Augustine $^{(86)}$, después de liberar distalmente el tendón del músculo semitendinoso, lo pasaba por la escotadura intercondílea y hacía un túnel tibial para fijarlo a su salida. Como en el caso de la técnica de Helfet ${ }^{(78)}$, los resultados dependían de una buena rehabilitación.

O’Donoghue ${ }^{(41,71)}$, en Oklahoma, popularizó la reparación del LCA en los EE. UU. En 29 deportistas, modificó la técnica de Hey-Groves y, aunque los resultados fueron buenos, también observó que el índice de éxitos no recomendaba la reconstrucción del LCA de forma rutinaria. Había que seleccionar a cada paciente e insistió en reparar los ligamentos de la rodilla en las 2 primeras semanas después de la lesión para obtener mejores resultados. En el caso de las lesiones crónicas con inestabilidad articular, recomendaba la técnica de Hey-Groves ${ }^{(34,54)}$ y, a diferencia de Helfet ${ }^{(78)}$ y Augustine ${ }^{(86)}$, dudaba de la reconstrucción dinámica con los tendones de los isquiotibiales. 


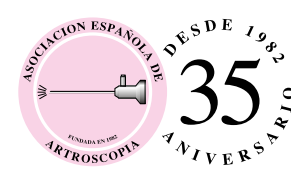

En 1959, Lindstrom ${ }^{(84)}$ publicó el mayor estudio hasta ese momento, con 34 casos de reparación del LCA utilizando el menisco, que era suturado, a través de perforaciones en el fémur y en la tibia. Walsh( ${ }^{(87)}$, en 1972, desechó esta técnica ante los malos resultados obtenidos.

Fue en la década de los sesenta cuando Kenneth Jones ${ }^{(88)}$ recomendó el injerto autólogo hueso-tendón-hueso (H-T-H) obtenido del tercio central del ligamento rotuliano que sería popularizada por William Clancy, de Madison, Wisconsin (EE.UU)(89), aunque la idea original había sido de Campbell(70) en 1936. En el H-T-H pasó de emplearse el tercio medial al injerto del tercio central. Clancy ${ }^{(89)}$ inicialmente la combinaba con técnicas extraarticulares de refuerzo, hasta que O'Brien et al. ${ }^{\left({ }^{0}\right)}$ demostraron que no eran necesarias. Clancy ${ }^{(91)}$, en 1963, defendió la técnica propuesta por Jones ${ }^{(88)}$ pero extrayendo el tercio central del ligamento vascularizado, es decir, con el tejido adiposo subyacente para mejorar su integración $n^{(77,84,87,88,92)}$.

Brückner ${ }^{(93)}$, de Rostock (República Democrática de Alemania), en 1966, introdujo la utilización de la porción interna del ligamento rotuliano que pasaba por un túnel tibial y otro femoral y, como la plastia solía quedar corta, la suturaba por el extremo femoral suspendida de un botón externo en el cóndilo femoral ${ }^{(94)}$. Años después, Enjar Eriksson ${ }^{(95)}$, de Estocolmo, Suecia, en 1976, apoyado en una idea de Broström ${ }^{(96)}$, mejoraba la técnica presentando una serie de 72 casos, con buenos resultados en el $80 \%$ de los mismos.

Una de las técnicas extraarticulares más utilizadas por aquellos años, empleada todavía hoy, fue la propuesta de Marcel Lemaire, en Parisis ${ }^{(97,98)}$, quien basado en la idea de que la función del LCA es principalmente mantener la estabilidad rotatoria, separó una tira de fascia lata y la pasó por debajo del ligamento lateral externo (LLE) para introducirla por un túnel, en la parte posterior del cóndilo externo, la sacaba de nuevo para suturarla en su origen en el tubérculo de Gerdy.

Nicholas y Minkoff(${ }^{(99)}$ reorientaron la cintilla iliotibial desinsertada distalmente con un bloque óseo a través de la cápsula posterior a la porción anterointerna de la tibia, por delante de la espina tibial. La técnica, llamada del "cinco en uno", buscaba corregir la inestabilidad rotacional anteromedial e incluía una meniscectomía total interna, avance posterior e interno de la inserción del
LLI, avance distal y anterior de la cápsula posteromedial, adelantamiento de la parte posterior del músculo vasto medial y transferencia de la pata de ganso. Como se puede ver, al igual que Hughston(2), consideraron que el ángulo posterointerno era la llave para obtener buenos resultados. Estas técnicas, pasado el tiempo, tampoco obtuvieron los resultados esperados y Ellison et al. ${ }^{(100)}$ modificaron su técnica, asociando el adelantamiento del músculo biceps femoral para dar mayor estabilidad.

En 1979, Marshall et al. ${ }^{(43)}$ enrollaron la porción central del ligamento rotuliano, como si fuera un cigarrillo, con la fascia prerrotuliana y una tira central del tendón del músculo cuádriceps; un injerto largo que pasaba por un túnel tibial y era llevado, cruzando la articulación, hacia el cóndilo femoral posterolateral over-the-top, por un surco preparado previamente ${ }^{(101)}$. Kurt Franke ${ }^{(102)}$, en Berlín, en 1976, fue, siguiendo la técnica de Brückner ${ }^{(94)}$, el primero en describir un trasplante libre de una porción de ligamento rotuliano con hueso, tanto de la rótula como de la tibia. Su publicación es la primera en reflejar la experiencia de un injerto H-T-H libre y de larga evolución. A partir de este trabajo con la aportación de Erikson ${ }^{(95,103)}$ en Europa, Clancy la popularizó en los EE. UU. ${ }^{(89)}$. Woods et al. ${ }^{(104)}$ tomaban también una porción de hueso rotuliano para conseguir un contacto hueso-hueso en el túnel femoral y después obtenían la longitud suficiente para fijarla en la región supracondílea femoral lateral fijándolo con un hilo guía. Eriksson ${ }^{(103)}$, como Palmer ${ }^{(26)}$ previamente, utilizó una guía para conseguir un anclaje anatómico.

Walter Blauth ${ }^{(105)}$, en Kiel, en 1984, fue el primero en publicar la utilización del tendón cuadricipital en las deficiencias crónicas del LCA. El injerto lo obtenía distalmente con un trozo de hueso de forma triangular, mientras que la porción proximal la dividía en 2 fascículos que permitían una reconstrucción bifascicular; uno de los fascículos pasaba por un túnel femoral y el otro over-the-top del cóndilo femoral externo. John Fulkerson $^{(106)}$, en Farmington, Connecticut, fue el promotor de la plastia del tendón cuadricipital que, aunque puede ser de mejor calidad, nunca ha llegado a popularizarse. El tendón del músculo cuádriceps tiene la ventaja de ofrecer un mayor volumen e injertos más uniformes y, técnicamente, permite que un cirujano efectúe la extracción mientras que otro prepara los túneles en la tibia y el fémur(107). 
En 1968, Lam ${ }^{(108)}$ volvió a tomar el tercio interno del ligamento rotuliano, colocando la inserción tibial en una posición más anatómica, con un bloque de hueso y un tornillo interferencial. Además, giraba el injerto $360^{\circ}$ para simular la morfología helicoidal del LCA. En 1980, en un intento de efectuar una sola incisión, Jones ${ }^{(109)}$ describió la utilización de un tornillo percutáneo para fijar el hueso rotuliano en el túnel femoral. Ese mismo año, Slocum et al..$^{(110-112)}$ definieron la "inestabilidad rotatoria" de la rodilla como consecuencia de la lesión de las estructuras mediales y del LCA. Describieron una prueba para ayudar con el diagnóstico y desarrollaron su técnica con la transferencia de la pata de ganso para controlar la inestabilidad. Considerando que la rotación externa de la tibia era la causa principal de la sintomatología de la inestabilidad, pensaron que cambiando la acción de la pata de ganso, de flexora a rotadora interna, controlarían la inestabilidad ${ }^{(113)}$.

John Insall(114,115), basado en los trabajos previos de Ellison ${ }^{(100)}$, de Jeffrey Minkoff y de James Nicholas ${ }^{(99)}$, en el Lenox Hill Hospital, en 1971, utilizó una tira de la cintilla iliotibial intraarticular, fijándola en la cara anterior de la tibia (bone block iliotibial band transfer). Desinsertaba la parte central de la fascia lata con parte del tubérculo de Gerdy, lo rotaba por fuera del cóndilo externo del fémur, over-the-top, y lo pasaba por un túnel para fijarlo por delante, en la cara anterior de la meseta tibial, aunque reconociendo que reconstruir la anatomía del LCA es una tarea imposible.

Brant Lipscomb et al.(116), de Nashville, fue de los primeros en utilizar los dos tendones de la pata de ganso y, 6 años más tarde, Marc Friedman ${ }^{(117)}$, en Los Ángeles, a la vista de los buenos resultados, utilizó la técnica de Lipscomb por artroscopia. Zarins y Rowe ${ }^{(118)}$, siguiendo los trabajos señalados de Macey ${ }^{(62)}$, popularizaron la técnica al publicar sus resultados con tendones de la pata de ganso.

En los años setenta, proliferaron los trabajos experimentales y conceptuales sobre el LCA y su reparación. La reconstrucción del LCA con el ligamento rotuliano es la técnica más frecuente y está considerada como la referencia en la cirugía del LCA ${ }^{(114,119-121)}$.

En 1974, Noyes et al. ${ }^{(122)}$ analizaron en una máquina de ensayos la resistencia del LCA en primates (Macaca mulatta) -un trabajo pionero que sirvió de referencia- y vieron que el complejo
$\mathrm{H}-\mathrm{T}-\mathrm{H}$ fallaba con altas cargas y con una elongación importante del ligamento. Además, señalaron que la rotura se producía por una avulsión tibial cuando el ensayo se hacía a baja velocidad y por disrupción de las fibras del ligamento cuando la velocidad era alta. También Clancy et al. (123) $^{(2)}$ presentaron, en 1981, el trabajo experimental de la reconstrucción de ambos ligamentos cruzados en Macaca rhesus.

En los años ochenta en los pacientes activos y, sobre todo, deportistas de alta competición se recomendaba combinar una técnica extraarticular con otra intraarticular (29,76,84,85,103,124-126). Según Marín et al. ${ }^{(126)}$, desaparecía el pivot-shift aunque persistía un cajón neutro o rotatorio externo con la rodilla a $90^{\circ}$ de flexión. Bray et al. ${ }^{(127)}$ revisaron, con más de 6 años de seguimiento, la evolución de 47 pacientes, 18 de ellos operados según la técnica de Maclntosh y los 29 restantes, asociando una reparación intraarticular del LCA con una plastia sintética de Dracon ${ }^{\circledR}$. No vieron diferencias entre ambos grupos. Se mostraron satisfechos con los resultados, aunque el grupo con la plastia sintética presentó mayor número de complicaciones y ambos grupos mostraron un deterioro a partir del tercer año de la cirugía.

Muchos cirujanos tenían claro que la rotura del LCA producía una inestabilidad articular y las técnicas disponibles ofrecían garantías suficientes para solucionarlo. Poco a poco, fueron apareciendo técnicas para reparar la inestabilidad rotacional y prevenir la subluxación de la tibia, manteniendo la rodilla en una posición que reducía la rotación interna, transfiriendo los tendones de la pata de ganso, la retracción capsular o el avance del LLI a una posición más proximal y posterior.

Todos los esfuerzos se encaminaron a corregir la inestabilidad residual de la rodilla y, así, McMaster et al. ${ }^{(63)}$ utilizaron el tendón del músculo recto interno. Cho et al. ${ }^{(128)}$ y Lipscomb et al. ${ }^{(112)}$ publicaron sus técnicas con el tendón del músculo semitendinoso, mientras que Horne y Parsons ${ }^{(129)}$ modificaron el procedimiento pasando el injerto a través de la cápsula posterior y over-the-top del cóndilo femoral lateral, una ruta más anatómica descrita inicialmente por Maclntosh. Collins et al. ${ }^{(130)}$ y Tillberg(131) volvieron a utilizar el menisco como injerto, demostrando mejores resultados que los ofrecidos por Walsh ${ }^{(87)}$.

En esta época todavía no se había abandonado la sutura primaria del LCA roto y Feagin(132), en 


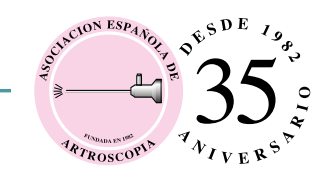

1976, sorprendió con sus entusiásticos resultados de la sutura del LCA en cadetes de la Academia Militar de West Point, en un congreso de la American Academy of Orthopaedic Surgeons (AAOS). Simultáneamente, Maclntosh describió sus buenos resultados de la reparación primaria suturando el ligamento roto, con una técnica que definió como over-the-top y que, modificada por John Marshal(43), se convirtió en el tratamiento de elección. Pero el propio Marshall ${ }^{(43,133)}$ la abandonó, pasando a efectuar reconstrucciones con fascia lata. Siete años después de su presentación, Feagin ${ }^{(134)}$ reconoció que el $84 \%$ de los cadetes operados con sutura directa se encontraron inicialmente bien y volvieron a practicar deporte, pero a los 5 años presentaban una inestabilidad recurrente de su rodilla, dos tercios presentaban dolor y 17 tuvieron una nueva rotura con deterioro progresivo en su función. La conclusión era clara y fue el primero de una línea de trabajos posteriores ${ }^{(135,136)}$ que demostraron la mala evolución de esta técnica.

La cinta iliotibial se hizo más popular para corregir las inestabilidades anterolaterales o combinadas. Losee et al. ${ }^{(137)}$ liberaban la cintilla proximalmente, pasando por un túnel extracapsular, por debajo del músculo gemelo externo, a través del cóndilo femoral lateral, de delante atrás y, después, de nuevo, hacia delante, por debajo del LLE, hasta llegar al tubérculo de Gerdy.

Tanto Maclntosh ${ }^{(138)}$ como Losee ${ }^{(137)}$ desinsertaban la porción proximal de la cintilla. Por su parte, Ellison ${ }^{(139)}$ describió una reconstrucción "dinámica" y para ello desinsertaba la cinta distal y rotando el injerto lo pasaba por debajo del LLE, considerando que la tensión provocada por el tensor de la fascia lata estabiliza el compartimento externo de la rodilla ${ }^{(140)}$ y así controlar la subluxación anterior del platillo tibial externo en extensión, en ausencia del LCA. Sin embargo, una gran mayoría de las rodillas operadas no obtenían buenos resultados ${ }^{(141)}$.

Unverferth y Bagenstose, en 1979(142), combinaron la técnica de Ellison ${ }^{(100,139)}$ con una capsulorrafia anteromedial, una transferencia de la pata de ganso y un avance parcial del músculo bíceps femoral. Youmans et al. ${ }^{(135)}$, además, combinaron una reconstrucción extraarticular medial y lateral. Andrews $^{(144,145)}$ defendía la importancia de la isometría y pensaba en una reconstrucción funcional, tanto en flexión como en extensión. Sacaba 2 tiras de la cinta iliotibial y las aseguraba, extraarticularmente, en el cóndilo femoral externo. Este procedimiento, aunque reducía el pivot-shift, no era funcional y perdía consistencia con el tiempo.

Galway y Maclntosh ${ }^{(143)}$ y otros $^{(144-148)}$ dieron a conocer el fenómeno del pivot-shift que Hughston et al.(147,148) incorporaron a su teoría de la inestabilidad rotacional, también llamada "inestabilidad rotacional anteroexterna", atribuyendo el fenómeno a la rotura de la cápsula externa que aumenta con la lesión del LCA. Torg et al. ${ }^{(23)}$ describieron la prueba de Lachman y demostraron su superioridad biomecánica frente a la prueba del cajón anterior.

MacIntosh ${ }^{(138,149)}$, en el Toronto General Hospital, describió la reconstrucción intraarticular con una tira obtenida de la cintilla iliotibial, conocida como técnica Maclntosh-1, que Andrews y Sanders ${ }^{(144,145)}$ modificaron, añadiendo su concepto de isometría, ganando mucha popularidad. La isometría pretendía mantener el injerto a la misma tensión en flexión y en extensión de la rodilla. Los resultados fueron inicialmente espectaculares, si bien se deterioraban con el tiempo. Esto hizo que Macintosh cambiase su técnica pasando el injerto intraarticular por un túnel tibial, conocida como Maclntosh-2, y siguió modificando su técnica utilizando injerto de tendón cuadricipital. El extremo proximal pasaba por la escotadura y lo aseguraba en la cara externa del fémur, conocido como quadriceps patellar tendon over-the-top o MacIntosh-3. No era una técnica isométrica y la propia delgadez del injerto provocaba, al cabo del tiempo, fallos. Para evitarlo, aumentó el grosor con una cinta de tendón cuadricipital (técnica de Marshall-Maclntosh) y también con material sintético ${ }^{(150)}$. Curiosamente, a pesar de la importancia de las técnicas de Maclntosh, son pocos los trabajos originales disponibles de su autor.

Amirault et al. ${ }^{(138)}$ y Ostermann et al. ${ }^{(149)}$ consideraron que las técnicas extraarticulares, como la de MacIntosh, eran la alternativa para prevenir un pivote central. Sin embargo, para Amis y Scammell(151), en las roturas aisladas del LCA no están justificadas y, si se hace una técnica intraarticular correcta, es innecesario acompañarla de métodos extraarticulares. Tampoco Jensen et al. ${ }^{(152)}$ ni Strum et al. ${ }^{(153)}$ encontraron diferencias al añadir un refuerzo extraarticular a una técnica intraarticular(154), combinación que tiende a producir grandes cajones anteriores, comprometiendo la estabilidad 
articular. O'Brien et al. ${ }^{\left({ }^{0}\right)}$ señalaron que, además de no aportar ninguna ventaja mecánica, estos pacientes presentan un $40 \%$ de sintomatología residual como consecuencia de la técnica extraarticular.

Kennedy et al. ${ }^{(140)}$ recomendaron ante una lesión aguda del LCA con arrancamiento femoral o tibial la reparación, pero cuando la rotura estaba en la porción media del ligamento se debían resecar los extremos. Si la lesión del LCA se asociaba con daños capsulares y de los ligamentos colaterales, consideraban la rotura del LCA secundaria. Además, cuando en una artrotomía se apreciaba una inestabilidad rotatoria externa asociada a la rotura del LCA, recomendaban una transposición de los tendones de la pata de ganso ${ }^{(111)}$ pero sabiendo que la transposición podría agravarla.

El cajón anterior positivo como prueba clara de rotura del LCA se había asumido desde la publicación de Palmer ${ }^{(26)}$, en 1938, aunque no faltaron ciertas reticencias con esta prueba ${ }^{(155-157)}$. Por su parte, Hughston et al. ${ }^{(147,148)}$ propusieron una clasificación de las inestabilidades de los ligamentos de la rodilla correlacionando con la clínica y señalando que un cajón anterior no es patognomónico de rotura del LCA. El cajón anterior aumenta cuando se asocia la rotura del LCA con una lesión del ligamento oblicuo posterior. Hughston et al. (147) escribieron que en "200 artrotomías observando un LCA normal, no vimos ni un solo LCA tenso a $90^{\circ}$ de flexión con el pie apoyado sobre la mesa de operaciones" y terminan, "según nuestras observaciones clínicas, anatómicas y quirúrgicas del LCA, nuestra impresión es que la función más importante del LCA es la prevención de la hiperextensión o del recurvatum. También podría actuar como guía en el mecanismo de rotación durante la extensión de la rodilla".

Toda cirugía intraarticular del LCA precisaba efectuar una artrotomía causando alteraciones de los elementos propioceptores de la cápsula articular, hasta que Rosenberg y Rasmunsen ${ }^{(158)}$, en 1984, describieron una técnica endoscópica, aprovechando el artroscopio diseñado por Watanabe e Ikeuchi(159-164), hoy perfectamente establecida, utilizada en la mayoría de los centros, que disminuye las complicaciones propias de la técnica y el tiempo de recuperación $n^{(6,44,81,165)}$.

Karl Ludloff(166), en 1927, señaló que la reconstrucción del LCA exige la colocación de 2 fascículos independientes. Karl Viernstein y Werner Keyl, en Múnich, fueron de los primeros en reconstruir el LCA con un doble fascículo desinsertando el tendón del músculo semitendinoso y del músculo recto interno proximales, en $1973^{(167)}$. Los tendones se rotaban y pasaban por un túnel tibial común y 2 túneles femorales separados y suturando uno contra otro a su salida. En 1983 apareció la primera publicación en inglés con la técnica del doble fascículo, William Mott ${ }^{(168)}$, de Jackson, Wyoming (EE.UU.), realizaba un doble túnel en tibia y fémur, por donde pasaba el injerto de semitendinoso con la técnica llamada STAR (semitendinosus anatomic reconstruction"), que sería seguida por Walter Blauth ${ }^{(105)}$, de Kiel, Alemania. En 1990, Jean Louis Meystre ${ }^{(169)}$, en Lausana, Suiza, publicó un $77 \%$ de buenos y excelentes resultados con una plastia del tendón del músculo semitendinoso y un doble túnel en la tibia y un único túnel en el fémur.

\section{La isometría}

Hemos citado la isometría como una de las bases del tratamiento quirúrgico del LCA ${ }^{(100,139)}$. El cirujano cuando reconstruye el LCA intenta controlar la tensión del injerto, de una forma subjetiva, al mismo tiempo que lo fija. Normalmente se fija el injerto de ligamento rotuliano en el túnel femoral y se aplica entonces la tensión en sentido distal anclándolo, posteriormente, en la tibia. El grado de flexión articular y la cantidad de tensión aplicada sobre el injerto son aspectos que se efectúan de forma empírica. Si el injerto se queda "muy suelto", no desaparecerá la laxitud articular anormal en sentido anteroposterior. Conseguir esta estabilidad y eliminar el pivot-shift son los indicadores de un buen resultado quirúrgico. Si, por el contrario, el injerto queda "muy tenso", se producirán alteraciones en el movimiento articular y la revascularización se verá afectada ${ }^{(170)}$.

La fijación correcta e isométrica del injerto es el factor técnico más importante para obtener buenos resultados ${ }^{(44,171-178)}$. Como han señalado Shino et al. ${ }^{(179)}$, un injerto $\mathrm{H}-\mathrm{T}-\mathrm{H}$ requiere una perfecta adaptación entre el grosor del túnel y el del injerto óseo.

En los años sesenta, la premisa era que la reconstrucción del LCA debe ser isométrica(108,180,181) y conseguir un arco de movilidad completo sin provocar la elongación ni la deformación plástica del 


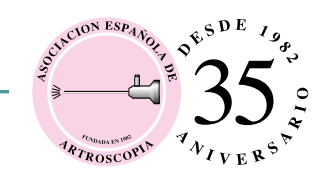

ligamento. Esto siempre ha resultado complicado cuando las plastias son cilíndricas, con unos túneles no siempre fáciles de orientar. El punto de inserción de las fibras neutras es conocido como lugar isométrico. Las fibras que se insertan por delante de la fibra neutra en la tibia se insertan posteriores en el fémur y viceversa ${ }^{(122)}$. Esta disposición minimiza los cambios de longitud durante la flexión de las fibras no isométricas.

En 1974, Artmann y Wirth ${ }^{(182)}$ definieron el punto isométrico del origen del LCA, recomendando perforar el túnel femoral en la porción posterosuperior de la huella anatómica, cerca del overthe-top. El túnel en la tibia es más crítico y, como ayuda orientativa, recomendaron que en la reconstrucción del LCA se debe procurar sustituir al fascículo anteromedial(182).

Yoshiya et al.(183) efectuaron un trabajo experimental en perros, sustituyendo el LCA por un injerto de tercio central de ligamento rotuliano a diferentes tensiones. Observaron que una tensión excesiva del injerto conllevaba cambios importantes en la histología del ligamento produciendo una degeneración mixoide focal y pérdida del paralelismo de las fibras de colágeno, demostrando una pobre vascularización con la microangiografía. Utilizando modelos experimentales, se han estudiado los cambios de longitud del LCA durante el arco completo de movimiento, siendo de 3 a 6 $\mathrm{mm}^{(184-186)}$. Estos cambios de longitud rompen con el modelo de rodilla formada por 4 barras constituidas por los 2 ligamentos cruzados, el fémur y la tibia, y se asume que una fibra neutra en cada ligamento permanece con su longitud constante, isométrica, durante el arco de flexión. El centro instantáneo articular, el eje de flexión en algunos puntos, es la intersección de estas fibras neutras. Las fibras que quedan por delante de este eje se alargan durante la flexión, mientras que las que quedan por detrás se acortan ${ }^{(22)}$. Para Hefzy y Grood $^{(171,173)}$, las fibras del LCA con inserción femoral posterior son más largas durante la extensión y más cortas durante la flexión, mientras que las fibras situadas en la porción media tienen la misma longitud en flexión que en extensión. Por su parte, Segal et al. ${ }^{(187)}$ consideraron que el LCA está torsionado, de forma que las fibras anteriores en la tibia son las fibras mediales en el fémur.

Pero también hay publicaciones que consideran que es todo el LCA el que está en tensión durante la extensión $\mathrm{n}^{(19,20,56,147,188-190)}$ o es solo la por- ción posteroexterna del LCA la que está en tensión durante la extensión de la articulación de la rodilla(21,191,192) y la anteromedial durante la flexión ${ }^{(20,21,133,191,192)}$. Palmer, en 1938 ${ }^{(26)}$, invirtió las porciones y consideraba que durante la extensión estaban en tensión las fibras anteromediales del LCA mientras que durante la flexión eran las fibras posterolaterales. Dawkins y Amis $^{(193)}$ dividieron el LCA en 3 porciones, sosteniendo que durante la extensión articular son las porciones anteromedial y posterolateral las que están en tensión y la parte central del LCA durante la flexión. Según Walker et al.(194), las fibras anteriores del LCA mantienen una longitud constante durante la flexión, mientras que las fibras posteriores disminuyen un $15 \%$ en su longitud. Por el contrario, las fibras posteriores del LCP permanecen con un $10 \%$ de su longitud inicial, pero hacia los $30^{\circ}$ de flexión las fibras anteriores comienzan a tensarse alcanzando un $25 \%$ de su longitud inicial a los $120^{\circ}$ de flexión.

Lo que aclararon estos trabajos fue que la mayoría de las fibras del LCA no son isométricas ${ }^{(195)}$ y por eso pueden contribuir a la estabilidad rotatoria cuando la rodilla se acerca a la extensión completa. Friederich y O'Brien ${ }^{(195)}$ hablaron de la "isometría funcional" al considerar que solo un pequeño número de fibras son realmente isométricas. Las fibras más isométricas son las primeras en tensarse, las restantes están dispuestas de manera que se van reclutando progresivamente, conforme la rodilla se acerca a la extensión. Por esto, Müller(124) introdujo el concepto de "anatometría", un compromiso entre isometría y posición anatómica de la plastia y deja de hablar de punto de isometría para citar la "línea de transición" que debe representar el borde anterior de la plastia, ya que las fibras de la plastia que se encuentran por delante de esta línea estarán expuestas a una elongación no fisiológica durante la flexión.

No es de extrañar que durante una época larga fueran apareciendo guías para facilitar la colocación de la plastia de forma correcta y homogénea; las primeras fueron las de Palmer ${ }^{(26)}$, con Soli Lam ${ }^{(108)}$, en Londres, Enjar Eriksson ${ }^{(103)}$, George Hewson ${ }^{(196)}$. En España, Mikel Sánchez patentó su guía que tuvo una gran difusión internacional. Además, Dale Daniel et al.(197), en San Diego, desarrollaron el KT-1000 Knee Ligament Arthrometer ${ }^{\circledR}$ y también el Isometer ${ }^{\circledR}$, en 1986. 


\section{Los ligamentos sintéticos y aloinjertos}

No podemos olvidar el interés que despertó la utilización de nuevas prótesis sintéticas para la sustitución del LCA, aunque los resultados a corto plazo demostraron su fracaso y no se ha vuelto a intentar en los últimos años ${ }^{(198,199)}$. Conviene señalar que fue Lange el primero en probar los "ligamentos artificiales" de seda ${ }^{(4)}$. Con los años se han probado fibras de polietileno y poliéster de alto rendimiento, adecuados a la anatomía del LCA, por sus propiedades mecánicas; se han publicado estudios con distintos materiales como nailon(200), ácido poliglicólico trenzado(201), Dacron ${ }^{\circledR(201)}$, polietileno Poliflex ${ }^{\circledR(198,202)}$, polietileno tereftalato (ligamento Leeds-Keio $\left.{ }^{\circledR}\right)^{(203,204)}$, Trevira ${ }^{\circledR(205)}$, politetrafluoretileno (Gore-Tex $\left.{ }^{\circledR}\right)^{(206,207)}$, polipropileno (Kennedy-Lad $\left.{ }^{\circledR}\right)^{(202,208)}$ y fibra de carbono ${ }^{(209,210)}$. Sin embargo, los implantes sintéticos se deforman plásticamente y se deforman permanentemente con la mitad de tensión que el LCA original(211,212).

El interés en la utilización de nuevas prótesis sintéticas para la sustitución del LCA es hoy experimental. La prótesis ideal debe proveer una función biológica adecuada, ser biocompatible y permitir el desarrollo y el crecimiento de colágeno en el interior de la prótesis, lo cual contribuye al refuerzo del ligamento y a su estabilidad y durabilidad durante largo tiempo ${ }^{(213)}$. Sin embargo, es necesaria una nueva generación de ligamentos sintéticos que combinen las ventajas de los materiales sintéticos (alta resistencia, fácil fabricación y almacenamiento) y los injertos biológicos (biocompatibilidad y crecimiento tisular)(214). El colágeno, por sus características físicas, químicas y biológicas, es un material que puede ser interesante para el desarrollo de prótesis ligamentosas, al ser el mayor componente extracelular del $L_{C A}^{(215)}$ que puede ser procesado en fibras reabsorbibles de alta resistencia, con diámetros semejantes al del LCA. El colágeno es biocompatible sin presentar problemas antigénicos y, además, es quimiotáctico para los fibroblastos y otras células que aparecen en el tejido de reparación ${ }^{(214)}$.

Otro desarrollo completamente distinto han sido los tendones alogénicos que se han utilizado con éxito en la reconstrucción de ligamentos de la articulación de la rodilla y otras articulaciones ${ }^{(216-221)}$. Presentan ventajas ${ }^{(222-226)}$, ya que no alteran o suprimen los tendones o fascias del propio paciente, el tiempo quirúrgico es más corto, existe la posibilidad de elegir el tamaño más adecuado entre un amplio arsenal de ligamentos o tendones, la técnica es menos agresiva, se pueden utilizar grandes injertos sin producir alteraciones funcionales, también se puede utilizar ligamento rotuliano que presenta mejores condiciones mecánicas que el tendón del músculo semitendinoso, disminuyen las rigideces de rodilla, permiten la reconstrucción ligamentosa múltiple, son injertos mecánicamente resistentes y, sobre todo, es la indicación en pacientes que han sido sometidos a revisiones del LCA con plastias autólogas anteriores. Los aloinjertos en la cirugía primaria del LCA están justificados cuando el paciente presenta problemas para la toma de injertos autólogos (tendinitis, secuelas de Osgood-Schlatter, etc.), si se necesita acortar el periodo de baja laboral o por motivos estéticos ${ }^{(227)}$.

Desde los años sesenta, diferentes autores demostraron experimentalmente que la incorporación de un aloinjerto tendinoso sigue la misma secuencia que un autoinjerto pero a menor velocidad ${ }^{(179,228-231)}$, aunque la aplicación clínica de los aloinjertos comenzó a finales de los años ochen$\mathrm{ta}^{(217,221)}$. Varios estudios demostraron que el mejor medio para reducir las propiedades antigénicas de los aloinjertos era su congelación profunda, a $-80^{\circ} \mathrm{C}^{(232-235)}$, aunque también se comercializaron los aloinjertos liofilizados ${ }^{(230,234)}$.

Sin embargo, también presentan problemas a tener en cuenta a la hora de su indicación, como son el riesgo de transmisión de enfermedades y las dificultades propias de su almacenamiento y manejo. Como señalan Co et al.(236), el fallo en la recuperación de la autorregulación propioceptiva puede producir un estiramiento excesivo del injerto y la consiguiente laxitud articular que se evita con los autoinjertos. En la revisión efectuada por Shino et al. ${ }^{(179)}$, las rodillas tratadas con aloinjertos muestran mejores resultados que las tratadas con autoinjertos comparando la estabilidad anterior de la rodilla y la resistencia del músculo cuádriceps.

Eugene Bircher ${ }^{(237,238)}$, en Arau, Suiza, pionero de la artroscopia, utilizó el tendón de canguro, cuyos resultados serían publicados por Micheli(239) 4 años más tarde. En 1984, Konsei Shino, de Osaka, Japón, presentó los resultados de los aloinjertos en 31 pacientes ${ }^{(179)}$; posteriormente, Richard Levitt(240), en Miami y también el grupo 


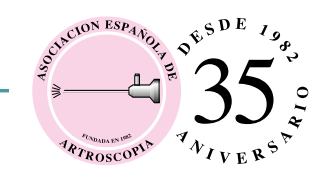

de Lieja (Bélgica), formado por Jacques Defrere y Anne Franckart ${ }^{(241)}$. En España, al mismo tiempo, Valentí(242) comenzó a desarrollar una de las mayores estadísticas mundiales.

\section{Los últimos años del siglo XX}

No es de extrañar que a partir de las experiencias anteriores el tratamiento de la rotura del LCA se enfocase para resolver la inestabilidad anterior y no tanto los daños estructurales, haciendo que todos los cirujanos buscasen modelos de reconstrucción cada vez más anatómicos. Todavía en 1985, Bonnel et al. ${ }^{(120)}$ recomendaban como cirugías más apropiadas la cuadricepsplastia de tipo Maclntosh, la plastia con refuerzo protésico de Dacron ${ }^{\circledR}$ o el trasplante libre vascularizado indicado por Clancy ${ }^{(91)}$.

Noyes et al. ${ }^{(122,219)}$ habian demostrado la eficacia mecánica del tercio central del ligamento rotuliano como plastia y que una porción de 14$15 \mathrm{~mm}$ de ancha era 1,5 veces más resistente que el LCA normal: además, como había demostrado experimentalmente Clancy $^{(91)}$, se producía una revascularización del injerto y, por entonces, la artroscopia diagnóstica había permitido ver que la sinovial recubría a la plastia. También, la integración ósea de los tacos del injerto H-T-H permitía una integración rápida que evitaba los aflojamientos con el tiempo. Sin lugar a dudas, era el injerto ideal. Paterson y Trickey ${ }^{(243)}$ modificaron la técnica obteniendo el tercio central del ligamento rotuliano libre, lo pasaban por un túnel tibial y lo fijaban over-the-top en el cóndilo femoral externo, evitando perforar el fémur.

Es importante reseñar que a principios de los ochenta aparecieron las primeras evaluaciones de las cirugías utilizando escalas de valoración con parámetros objetivos y subjetivos para revisar con un criterio más homogéneo los resulta-

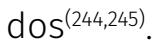

A finales de los años ochenta, comienza a ponerse en tela de juicio la idoneidad del tercio central del ligamento rotuliano como injerto ideal; se confundieron aspectos técnicos y subjetivos. Sin embargo, esto contribuyó a la mayor utilización de los tendones de la pata de ganso. Burks et al. ${ }^{(246)}$ consideraron que el tercio central del ligamento rotuliano producía alteraciones de las propiedades mecánicas del ligamento rotu- liano restante. También se señaló que no estaba indicado en pacientes que requerían trabajos en flexión o jugar con niños pequeños en el suelo ${ }^{(247)}$. La toma de injerto del ligamento rotuliano puede molestar, especialmente en la zona de la tuberosidad anterior de la tibia ${ }^{(248)}$ por el daño de las ramas infrarrotulianas del nervio safeno o de los ramos periósticos en la zona de toma de injerto o por la formación de un neuroma ${ }^{(249)}$.

Sin embargo, con la experiencia adquirida hasta ese momento, en la Universidad de Pittsburgh(226), entre 1985 y 1991, 2 cirujanos efectuaron 506 reconstrucciones de LCA, con 324 autoinjertos y 45 aloinjertos y el injerto $\mathrm{H}-\mathrm{T}$-H había sido utilizado en el $90 \%$ de los casos. Otros estudios comparativos entre auto- y aloinjertos no han demostrado diferencias entre estos 2 tipos de injertos y esto ha impulsado a algunos autores a utilizarlo de forma rutinaria. Pero Cosgarea et al.(248), en 191 reconstrucciones del LCA, utilizando injerto autólogo de la parte central del ligamento rotuliano, demostraron un $12 \%$ de artrofibrosis que requirieron cirugías posteriores con resultados poco satisfactorios. La incidencia de este tipo de complicación se estableció, a principios de los años noventa, en el $4 \%$, en 3 series con rotura única del LCA ${ }^{(249,250)}$, pero aumentaba al $23 \%$ en pacientes con rotura del LCA combinada con rotura del LLI o al 35\% en un grupo de pacientes con rotura aguda y reparación inmediata.

Actualmente, el tercio central del tendón rotuliano autólogo sigue siendo el injerto más utilizado para la reparación del LCA y el patrón con el que deben compararse los demás injertos. Efectivamente, este injerto fue el preferido por el $79,1 \%$ de los 249 cirujanos que participaron en una encuesta realizada en 2003 sobre el tratamiento de las lesiones del LCA ${ }^{(251)}$. En un estudio similar llevado a cabo por la Asociación Española de Artroscopia, el porcentaje de injertos rotulianos autólogos usados en nuestro país fue del $71 \%{ }^{(252)}$. Un grupo de cirujanos australianos de rodilla ${ }^{(253)}$ revelaron que utilizaban autoinjerto, de ligamento rotuliano o de la pata de ganso, en el 58\% según las circunstancias. El resto utilizaban o solo ligamento rotuliano o solo pata de ganso. Miembros del Grupo de Estudio del LCA ${ }^{(254)}$ presentaron otra perspectiva; el 73\% eligieron ligamento rotuliano, el 23\% pata de ganso y un 4\% "otros", como los aloinjertos. Bach et al. ${ }^{(255)}$ señalan el uso de aloinjertos ha ido en aumento; entre 1986 y 1996, la reconstrucción primaria con 
aloinjertos era del 2\%; entre 1996 y 2001, aumentó al $14 \%$; y entre 2002 y 2005 , alcanzó el $36 \%$.

Bray et al. ${ }^{(127)}$, en 1988, planteaban 4 aspectos a considerar en la cirugía del LCA. El primero seguía siendo la indicación de la técnica intra- o extraarticular y señala el curioso comentario de Helfet ${ }^{(78)}$, para quien "las técnicas intraarticulares no tienen una indicación especial o principal". Otro punto es el tiempo de evolución en los estudios y critican aquellos trabajos que no tienen, al menos, un seguimiento de 5 años. El tercer aspecto era la falta de correlación entre los signos clínicos, la prueba del cajón anterior y un Lachman positivo, con los resultados funcionales. Por último, aunque era conocida la asociación de la rotura del LCA con otras estructuras articulares, no se sabía cómo repercutía en la evolución de la reparación.

Otro aspecto que se planteó por aquellos años era conocer la tensión adecuada del injerto al mismo tiempo que se fijaba. Una idea quedaba clara, la fijación correcta e isométrica del injerto era el factor técnico importante para obtener buenos resultados. Además, la colocación de un injerto H-T-H exigía una perfecta adaptación entre el grosor del túnel y el del hueso para evitar zonas sin contacto entre las estructuras ${ }^{(220)}$. Todos estos aspectos contribuían no solo a la integración de la plastia, también al proceso de transformación de un tendón en un ligamento, lo que se dio en llamar "ligamentización"(256).

Hoy, años después, disponemos de mejores conocimientos y mayor experiencia y, sobre todo, de un desarrollo técnico que ha permitido efectuar la cirugía articular de la rodilla por vía artroscópica. Se han unificado criterios y prácticamente las cirugías de la reparación del LCA se pueden dividir por el tipo de injerto, en autólogos o aloinjertos. Dentro de los autoinjertos, entre los que utilizan $\mathrm{H}-\mathrm{T}-\mathrm{H}$ o tendones de la pata de ganso y, además, entre una gran mayoría que se inclina por la técnica monofascicular y otro grupo, menos numeroso, que defiende la técnica bifascicular.

Además, no podemos olvidar lo que han supuesto las técnicas de visualización directa como es la artroscopia y, sobre todo para el diagnóstico, la resonancia magnética y, en menor medida, la tomografía computarizada y la ecografía. La imagen anatómica ha ganado en detrimento de la exploración física y las maniobras clásicas.

Pero todavía quedan muchos aspectos por conocer y desarrollar. Queremos citar algunos de ellos para finalizar. Vemos interés en acelerar el tiempo necesario para la integración de la plastia, la tensión más adecuada, el grosor tanto del túnel como de la plastia, las posiciones e inclinaciones óptimas para cada paciente y, sobre todo, poder distinguir entre los pacientes candidatos a cirugía y aquellos que podrían vivir en su actividad diaria con un LCA roto compensado con las estructuras musculotendinosas restantes. Sea como fuere, poco a poco, se irán aclarando estos y otros aspectos, sin olvidar que una técnica quirúrgica hoy tan frecuente y "sencilla" ha tenido una historia apasionante de ideas y comprobaciones, de ilusiones y decepciones, de suposiciones y evaluaciones, de modas y abandonos, no siempre con criterios científicos, que conviene conocer y no olvidar. No hemos hablado de algunas publicaciones y cirujanos de los últimos años; nos hemos detenido antes de empezar el siglo XXI pues hoy se sigue escribiendo la continuación de esta historia.

\section{Bibliografía}

1. Anetzberger H, Müller-Gerbl M, Scherer MA et al. Veränderung der subchondralen Mineralisierung nach Rekonstruktion des vorderen Kreuzbands beim Schaf. Unfallchirurg. 1994;97:655-60.

2. Hughston JC, Eilers AF. The role of posterior oblique ligament in repairs of acute medial (collateral) ligament tears of the knee. J Bone Joint Surg (Am). 1973;55-A:923-40.

3. Inoue M, McGurk E, Hollis JM, Woo SLY. Treatment of the medial collateral ligament injury. Am J Sports Med. 1987;15:15-21.

4. Di Matteo B, Tarabella V, Filardo G, Tomba P, Viganò A, Marcacci $M$, et al. Knee multi-ligament reconstruction: a historical note on the fundamental landmarks. Knee Surg Sports Traumatol Arthrosc. 2015;23:2773-9.

5. Forriol F, Ripoll P. La reparación del ligamento cruzado anterior: solución de un problema histórico en el siglo XX. Trauma (Mapfre). 2012;23:29-47.

6. Pässler HH. The history of the cruciate ligaments: some forgotten (or unknown) facts from Europe. Knee Surg Sports Traumatol Arthrosc. 1993;1:13-6.

7. Schindler OS. Surgery for anterior cruciate ligament deficiency: a historical perspective. Knee Surg Sports Traumatol Arthrosc. 2012;20:5-47.

8. Weber W, Weber E. Mechanik des menschlichen Gehwerkzeuge. Gotinga: Dieterische Buchhandlung; 1836. 


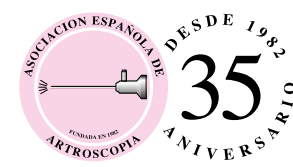

9. Langer, MK. Das Kniegelenk des Menschen. Dritter Beitrag zur vergleichenden Anatomie und Mechanik der Gelenke. Sitzungsberichte der Mathematisc-Naturwissenschaftlichen Classe der Kaiserlichen Akademie der Wissenschaften. 1858;32:99-112.

10. Dittel L. Über intraartikuläre Verletzungen am Knie. Wiener Med Jahrbücher. 1876;1876:319-34.

11. Bonnet A. Traité des maladies des articulations. París: Bailliere; 1845.

12. Hönigschmied J. Leichenexperimente über die Zerreisung der Bänder im Kniegelenk. Dtsch Z Chir. 1983;36:587-620.

13. Segond PF. Recherches cliniques et expérimentales sur les épanchements sanguins du genou par entorse. Prog Med. 1879;16:297-421.

14. Von Meyer H. Mechanik des Kniegelenkes. Müller's Archiv. 1853;497-547.

15. Zuppinger H. Die aktive Flexion in umbelasteten Kniergelenk. Anatomische Hefte. Beiträge und Referate zur Anatomie und Entwicklungsgesichte. 1904;77:701-64.

16. Straßer H. Lehrbuch der Muskel- und Gelenkmechanik. Bd. III. Die untere Extremität. Berlín: Springer; 1917.

17. Fick R. Handbuch der Anatomie und Mechanik der Gelenke. 3. Spezielle Gelenk- und Muskelmechanik. Jena: Gustav Fischer; 1911.

18. Pfab B. Expeimentelle Studien zur Pathologie der Binnenverletzungen des Kniegelenkes. Dtsch Z Chir. 1927;205:258-64.

19. Brantigan OC; Vorshell AF. The mechanics of the ligaments and menisci of the knee joint. J Bone Joint Surg. 1941;23:44-66.

20. Abbott LC, Saunders JB, Bost FC, Anderson CE. Injuries to the ligaments of the knee joint. J Bone Joint Surg (Am). 1944;26-A:503-21.

21. Girgis FG, Marshall JL, Al Monajem ARS. The cruciate ligaments of the knee joint. Clin Orthop Rel Res. 1975;106:216-31.

22. Norwood LA, Cross MM. Anterior cruciate ligament: functional anatomy of its bundles in rotatory instabilities. Am J Sports Med. 1979;7:23-6.

23. Torg JS, Conrad W, Kalen V. Clinical diagnosis of anterior cruciate ligament instability in the athlete. Am J Sports Med. 1976;4:84-93.

24. Forriol F. Epónimos 6. Trauma (Mapfre). 2012;23:130-7.

25. Kennedy JC, Weinberg HW, Wilson AS. The anatomy and function of the anterior cruciate ligament: as determined by clinical and morphological studies. J Bone Joint Surg (Am). 1974;56-A:223-5.

26. Palmer I. On the injuries to the ligaments of the knee joint. Acta Orthop Scand. 1938;53(suppl):1-21.
27. Arnoczky SP, Rubin RM, Marshall. Microvasculature of the cruciate ligaments and its response to injury: an experimental study in the dog. J Bone Joint Surg (Am). 1979;61-A:1221-9.

28. Arnoczky SP. Anatomy of the anterior cruciate ligament. Clin Orthop Rel Res. 1983;172:19-25.

29. Trillat A, Rainaut JJ. Traitment des laxites ligamentaires du genou. Rev Chir Orthop. 1959;45:97-117.

30. Adams R. Abnormal conditions of the knee joint. En: Todd RB (ed.). Cyclopaedia of anatomy and physiology, vol. 3. Londres: Sherwood Gilbert\&Piper; 1847. pp. 48-78.

31. Stark J. Two cases of rupture of the crucial ligaments of the knee joint. Edimburg Med Surg J. 1850;74:267.

32. Robson AWM. Ruptured crucial ligaments and their repair by operation. Ann Surg. 1903;37:716.

33. Lange F. Über die Sehnenplastik. Verh Dtsch Orthop Ges. 1903;2:10-2.

34. Kirschner M. Über freie Sehnen und Fascientransplantation. Bruns Beitr kiln Chir. 1909;65:472-503.

35. Davis JS. The transplantation of free flaps of fascia. An experimental study. Ann Surg. 1911;54:734-8.

36. Bick EM. History and source book of orthopedic surgery. Nueva York: The Hospital of Joint Diseases; 1933.

37. Goetjes HP. Über Verletzungen der Ligamenta cruciate des Kniegelenks. Dtsch Z Chir. 1913;123:221-89.

38. Perthes G. Über die Wiederfestigung des abgerissenen vorderen Kreuzbandes im Kniegelenk. Zentralbl Chir. 1926;53:866-72.

39. Sandberg R, Balkfors B, Nilsson B, Westlin N. Operative versus non-operative treatment of recent injuries to the ligaments of the knee. A prospective randomized study. J Bone Joint Surg (Am). 1987;69-A:1120-6.

40. Jonash E. Das Kniegelenk. Berlín: de Gruyter; 1964.

41. O'Donoghue DH. An analysis of end results of surgical treatment of major injuries to ligaments of the knee. J Bone Joint Surg (Am). 1955;37-A:1-13.

42. Quigley TB. Injuries to the ligaments of the knee. Clin Orthop Rel Res. 1954;3:20-8.

43. Marshall JL, Warren RF, Wickiewicz TL, Reider B. The anterior cruciate ligament: a technique of repair and reconstruction. Clin Orthop Rel Res. 1979;143:97-106.

44. Noyes FR, Butler DL, Paulos LE, Grood ES. Intra-articular cruciate reconstructions. 1: Perspectives on graft strength, vascularization and immediate motion after replacement. Clin Orthop Rel Res. 1983;172:71-7.

45. Payr E. Der heutige Stand der Gelenkchirurgie. Arch klin Chir. 1927;148:404-521.

46. Wagner P. Islierte Ruptur des Ligamenta Cruciata [Tesis doctoral]. Leipzig: Verlag von FCW Vogel; 1913.

47. Giertz KH. Über freie Transplantation der fascia lata als Ersatz für Sehnen und Bänder. Dtsch Z Chir 1913;125:480-96. 
48. Hesse E. Über den Ersatz der Kreuzbanänder des Kniegelenkes durch freie Fascientransplantation. Verh Dtsch Ges Chir 1914:43:188-9.

49. Matti H. Ersatz der gerissen vorderen Kreuzbandes durch extrartikuläre freie Fascientransplantation. Munch Med Wschr. 1918;17:451-2.

50. Bennett GE. The use of fascia for the reinforcement of relaxed joints. Arch Surg. 1926;13:655-66.

51. Bertocchi C, Bianchetti M. Sull evoluzione degli innesti autoplastici transossei e transarticolari di fascia e di tendine con particolare riguardo alla riconstruzione dei legamenti crociati. Chir Org Mov. 1923;7.

52. Zanoli R. Fratture del ginocchio. Chir Org Mov. 1928;12:482.

53. Hey Groves EW. Operation for the repair of the crucial ligaments. Lancet. 1917;II:674.

54. Hey Groves EW. The crucial ligaments of the knee joint: their function, rupture, and operative treatment of the same. Br J Surg. 1920;7:505-15.

55. Smith $A$. The diagnosis and treatment of injuries of the crucial ligaments. Br J Surg. 1918;6:176-89.

56. Cubbins WR, Conley AH, Callahan JJ, SCuderi CS. A new method of operating for the repair of the ruptured crucial ligaments of the knee joint. Surg Gynecol Obstet. 1932;54:299-306.

57. Putti V. La riconstruzione dei ligamenti crociati del ginocchio. Chir Org Mov. 1920;4:96

58. Holzel P. Fall von Zerrissung beider Kreuzbander des linken Kniegelenks, geheilt durch Ersatz aus dem luxierten ausseren Meniskus. Munch Med Wochenschr. 1917;28.

59. Eikenbary CF. A suggested method for the repair of crucial ligaments of the knee. Surg Gynecol Obstet. 1927;45:93-4.

60 . Edwards $\mathrm{AH}$. Operative repair of cruciate ligaments in severe trauma of knee. Br J Surg. 1926;13:432-8.

61. Galeazzi R. La ricostituzione dei ligament crociati del ginocchio. Atti e Memorie della Societá Lombarda di Chirurgia. 1934;13:302-17.

62. Macey HB. A new operative procedure for repair of ruptured cruciate ligaments of the knee joint. Surg Gynecol Obstet. 1939;69:108-9.

63. McMaster HJ, Weinert CR, Scranton P. The diagnosis and management of isolated anterior cruciate tears. A preliminary report on reconstruction with the gracilis tendon. J Trauma. 1974;14:230-5.

64. D’Aubigné M. Les lesions de l'appareil ligamentaire du genou (55 cas operés). En: Reconstruction surgery and traumatology, vol. 4. Basilea: Karger; 1957. pp. 156-80.

65. Lange M. Tratado de cirugía ortopédica. Barcelona: Ed. Labor; 1968.

66. Galli H. Kniebandverletzungen. Munch Med Wschr. 1963;105:1066-72.
67. Gold E. Volständinger plastischer Ersatz des vorderen Kreuzbandes und funktionell-anatomische Wiederherstellung desselben. Dtsch Z Chir. 1928;213:120-6.

68. Wittek A. Kreuzbandersatz aus den lig patellae (nach zur Verth). Schweiz med Wschr. 1935;65:103.

69. Campbell WC. Repair of the ligaments of the knee. Surg Gynecol Obstet. 1936;62:964-96.

70. Campbell WC. Reconstruction of ligaments of the knee. Am J Surg. 1939;43:473-80.

71. O'Donoghue DH. A method for replacement of the anterior cruciate ligament of the knee. J Bone Joint Surg (Am). 1963;45-A:905-24.

72. Mauck HP. A new operative procedure for instability of the knee. J Bone Joint Surg (Am) 1936;18-A:984-90.

73. Cotton FJ, Morrison GM. Artificial ligaments at the knee: technique. New Eng J Med. 1934;210:1331.

74. Bosworth DM, Bosworth BM. Use of fascia lata to stabilize the knee in cases of ruptured cruciate ligaments. J Bone Joint Surg (Am). 1936;18-A:178-9.

75. Kennedy JC; Fowler PJ. Medial and anterior instability of the knee. J Bone Joint Surg (Am). 1971;53-A:1257-70.

76. Moragas Badia J. Lesiones ligamentosas recientes de la rodilla. Acta Ortop Traumatol Ibérica. 1956;4:204-14.

77. Hauser EDW. Extra-articular repair for ruptured collateral and cruciate ligaments. Surg Gynecol Obstet. 1947;84:339-45.

78. Helfet AJ. Function of the cruciate ligament of the knee joint. Lancet. 1948;1:665-7.

79. Lindemann K. Über den plastischen Ersatz der Kreuzbander durch gestielte Sehnenverplanzungen. Z Orthop. 1950;79:316.

80. Dutoit GT. Knee joint cruciate ligament substitution. The Lindemann (Heidelberg) operation. S Afr J Surg. 1967:5:25-30.

81. Ficat P. Reconstruction du ligament croisé antérieur. Rev Chir Orthop. 1972;58(suppl 1):85

82. Groulier P, Bonsignour JP, Verola J. Traitement des laxities chroniques du pivot central du genou par transplantation dynamique selon Lindemann et Ficat. Marseille Chir. 1976;102:41-8.

83. Bousquet G, Millon J, Bascoulergue G, Rhenter JL. Les reflections du ligament croisé antérieur par la plastie activo-passive du pivot neutral et des points d'angle. Rev Chir Orthop. 1980;66(suppl II):91-2.

84. Lindstrom N. Cruciate ligament plastics with meniscus. Acta Orthop Scand. 1959;29:150-1.

85. Ramón Soler R, Vilalta Bou C, Cebamanos Celma C, Vilaró Portet L. La intervención de Lindemann en el tratamiento de las laxitudes crónicas de la rodilla. Rev Ortop Traumatol. 1987;31-IB:41-5. 
86. Augustine RW. The unstable knee. Am J Surg 1956; 92:380-8

87. Walsh JJ Jr. Meniscal reconstruction of the anterior cruciate ligament. Clin Orthop Rel Res. 1972;89:171-7.

88. Jones KG. Reconstruction of the anterior cruciate ligament. A technique using the central one-third of the patellar ligament. J Bone Joint Surg (Am). 1963;45A:925-32.

89. Clancy WG, Nelson DA, Reider B, Narechania RG. Anterior cruciate ligament reconstruction using one-third of the patellar ligament, augmented by extra-articular tendon transfers. J Bone Joint Surg (Am). 1982;64-A:352-9.

90. O'Brien SJ, Warren RF, Paulov H, Panarello R, Wickiewicz T. Reconstruction of the chronically insufficient anterior cruciate ligament with the central third of the patellar ligament. J Bone Joint Surg (Am) 1991;73A:278-86.

91. Clancy W. Anterior cruciate ligament functional instability. Clin Orthop Rel Res. 1963;172:102-6.

92. Zaricynyj B. Reconstruction of the anterior cruciate ligament using free tendon graft. Am J Sport Med. 1983;11:164-76.

93. Brückner H. Eine neue Methode der Kreuzbandplastik. Chirurg. 1966;37:413-4.

94. Brückner H, Brückner H. Bandplastiken im Kniebereich nach dem "Baukastenprinzip". Zbl Chir. 1972;97:65.

95. Eriksson E. Sports injuries of the knee ligaments. their diagnosis, treatment, rehabilitation, and prevention. Med Sci Sports. 1976;8:133-44.

96. Broström L, Gillquist J, Liljedahl S-O, Lindvall N. Behanding av inveterad ruptur av främre korsbandet. Läkartidn. 1968;64:4479.

97. Lemaire M. Instabilité chronique du genou. Techniques et resultants des plasties ligamentaires en traumatology sportive. J Chir. 1975;110:281-94.

98. Lemaire M. Ruptures anciennes du ligament croisé antérieur. Frequence - clinique - traitement. J Chir. 1967;93:311-20.

99. Nicholas JA, Minkoff. The five-one reconstruction for anteromedial instability of the knee. J Bone Joint Surg (Am). 1973;55-A:899-922.

100. Ellison AE. The pathogenesis and treatment of anterolateral rotatory instability. Clin Orthop Rel Res. 1980;147:51-5.

101. Marshall JI, Warren RF, Wickiewicz TL. Primary surgical treatment of anterior cruciate ligament lesions. Am J Sports Med. 1982;10:103-7.

102. Franke K. Clinical experience in 130 cruciate ligament reconstructions. Orthop Clin North Am. 1976;7:191-3.

103. Eriksson E. Reconstruction of the anterior cruciate ligament. Orthop Clin North Am. 1976;7:167-79.
104. Woods GW, Homsy CA, Prewitt JM, Tullos HS. Proplast leader for use in cruciate ligament reconstruction. Am J Sports Med. 1979;7:314-20.

105. Blauth W. Die zweizügelige Ersatzplastik des vorderen Kreuzbandes aus der Quadricepssehne. Unfallheilk. 1984;87:45-51.

106. Fulkerson JP, Langeland R. An alternative cruciate reconstruction graft: the central quadriceps tendon. Arthroscopy. 1995;11:252-4.

107. Stäubli HU. The knee and the cruciate ligaments. En: Jakob R, Staubli HU (eds.). Nueva York: Springer-Verlag; 1992.

108. Lam SJS. Reconstruction of the anterior cruciate ligament using the Jones procedure and its Guy's Hospital modification. J Bone Joint Surg (Am). 1968;50-A:1213-24.

109. Jones KG. Results of use of the central one-third of the patellar ligament to compensate for anterior cruciate ligament deficiency. Clin Orthop Rel Res. 1980;147:39-44.

110. Slocum DB, Larson RL. Pes anserinus transplantation. A surgical procedure for control of rotatory instability of the knee. J Bone Joint Surg (Am). 1968;50-A:226-42.

111. Slocum DB, Larson RL James SL. Pes anserinus transplant: Impressions after a decade of experience. J Sports Med. 1974;2:123-69.

112. Slocum DB, James SL, Larson RL, Singer KM. Clinical test for anterolateral rotatory instability of the knee. Clin Orthop Rel Res. 1976;118:63-9.

113. Ritter MA, Leaming ES, McCarroll JR. Preliminary report on the Jones, Ellison, Slocum (JES) repair for symptomatic anterior cruciate deficient knee. Am J Sports Med. 1983;11:89-94.

114. Insall J, Joseph DM, Aglietti P, Campbell D. Bone blok iliotibial band transfer for anterior cruciate insufficiency. J Bone joint Surg (Am). 1981;63-A:560-9.

115. Insall JN. Surgery of the knee. Nueva York: Churchill Livingstone; 1984.

116. Lipscomb AB, Johnston RK, Snyder RB. The technique of cruciate ligament reconstruction. Am J Sport Med. 1981;9:77-81.

117. Friedman MJ. Arthroscopic semitendinosus (gracilis) reconstruction for anterior cruciate ligament deficiency. Tech Orthop. 1988;2:74-80.

118. Zarins B, Rowe CR. Combined anterior cruciate ligament reconstruction using semitendinosus tendon and iliotibial tract. J Bone Joint Surg (Am). 1986;68A:160-277.

119. Bonnel F, Jaeger JH, Mansat CH. Les laxités chroniques du genou. París: Ed. Masson, 1984.

120. Arnoczky SP, Tarvin GB, Marshall JL. ACL replacement using patellar tendons. J Bone Joint Surg (Am) 1982;64A:217-24. 
121. Alm A, Gillquist J. Reconstruction of the anterior cruciate ligament by using the medial third of the patellar ligament. Acta Chir Scand. 1974;140:289-96.

122. Noyes FR, De Lucas JL, Torvik PJ. Biomechanics of anterior cruciate ligament failure: An analysis of strainrate sensitivity and mechanisms of failure in primates. J Bone Joint Surg (Am). 1974;56-A:236-53.

123. Clancy WG, Narechania RG, Rosenberg TD, Gmeiner JG, Wisnefske DD, Lange TA. Anterior and posterior cruciate ligament reconstruction in rhesus monkeys-a histological, microangiographic, and biomechanical analysis. J Bone Joint Surg (Am). 1981;63-A:1270-84.

124. Müller W. The knee. Form, function and ligament reconstruction. Würzburg: Springer Verlag; 1983.

125. Naves J, Rubies P. La rodilla. Barcelona: Ed. Científico Médica; 1959. pp. 314-35.

126. Marín Navarro M, Fuster i Obregon S, Jimeno Urban F, Cabot Dalmau J. Laxitud combinada anteromedial anterolateral de la rodilla. Doble plastia extraarticular. Rev Ortop Traumatol. 1987;31।B:375-82.

127. Bray RC, Flanagan JP, Dandy DJ. Reconstruction for chronic anterior cruciate instability. A comparison of two methods after six years. J Bone Joint Surg (Br). 1988;70-B:100-5.

128. Cho KO. Reconstruction of the anterior cruciate ligament by semitendinosus tenodesis. J Bone Joint Surg (Am). 1975;57-A:608-12.

129. Horne JG, Parsons CJ. The anterior cruciate ligament: its anatomy and a new method of reconstruction. Can J Surg. 1977;20:214-20.

130. Collins HR, Hughston JC, DeHaven KE, Bergfeld JA, Evarts CM. The meniscus as a cruciate ligament substitute. Am J Sports Med. 1974;2:11-21.

131. Tillberg $B$. The late repair of torn cruciate ligaments using menisci. J Bone Joint Surg (Br). 1977;59-B:15-9.

132. Feagin JA, Curl WW. Isolated tear of the anterior cruciate ligament: 5-year follow-up study. Am J Sports Med. 1976;4:95-100.

133. Furman W, Marshall JL, Girgis FG. The anterior cruciate ligament. J Bone Joint Surg (Am). 1976;58-A:179-85.

134. Feagin JA, Blake WP. Postoperative evaluation and result recording in the anterior cruciate ligament reconstructed knee. Clin Orthop Rel Res. 1983;172:143-7.

135. Youmans WT. The so-called "isolated" anterior cruciate ligament tear or anterior cruciate ligament syndrome: a report of 32 cases with some observation on treatment, its effect on results. Am J Sports Med. 1978;6:26-30.

136. Engebretsen L, Benum P, Fasting O, Mфlster A, Strand T. A prospective, randomized study of three surgical techniques for treatment of acute ruptures of the anterior cruciate ligament. Am J Sports Med. 1990;18:585-90.
137. Losee RE, Johnson TR, Southwick WO. Anterior subluxation of the lateral tibial plateau: a diagnostic test and operative repair. J Bone Joint Surg (Am). 1978;60A:1015-30.

138. Amirault JD, Cameron JC, Macintosh DL, Marks P. Chronic anterior cruciate ligament deficiency. Long term results of Maclntosh lateral substitution reconstruction. J Bone Joint Surg (Br). 1988;70-B:622-4.

139. Ellison AE. Distal iliotibial band transfer for anterolateral rotatory instability of the knee. J Bone Joint Surg (Am). 1979;61-A:330-7.

140. Kennedy JC, Stewart R, Walker DM. Anterolateral rotatory instability of the knee joint. J Bone Joint Surg (Am). 1978;60-A:1031-9.

141. McCulloch P, Latterman C, Boland AL, Bach BR. An illustrated history of anterior cruciate ligament surgery. J Knee Surg. 2007;20:95-104.

142. Unverferth LJ, Bagenstose JE. Extra-articular reconstruction surgery for combined anterolateral-anteromedial rotatory instability. Am J Sports Med. 1979;7:34-9.

143. Galway HR, Maclntosh DL. The lateral pivot shift: a symptom and sign of anterior cruciate ligament insufficiency. Clin Orthop Rel Res. 1980;147:45-50.

144. Andrews JR, Sanders R. A “mini-reconstruction” technique in treating anterolateral rotatory instability (ALRI). Clin Orthop Rel Res. 1983;172:93-6.

145. Andrews JR, Sanders RA, Morin B. Surgical treatment of anterolateral rotatory instability. A follow-up study. Am J Sports Med. 1985;13:112-9.

146. Ireland J, Trickey EL. Maclntosh tenodesis for anterolateral instability of the knee. J Bone Joint Surg (Br). 1980;62-B:340-5.

147. Hughston JC, Andrews JR, Cross MJ, Moschi A. Classification of knee ligament instabilities. I. The medial compartment and cruciate ligaments. J Bone Joint Surg (Am). 1976;58 A:159-72.

148. Hughston JC, Andrews JR, Cross MJ, Moschi A. Classification of knee ligament instabilities. II. The lateral compartment. J Bone Joint Surg (Am). 1976;58 A:173-9.

149. Ostermann K, Kujala UM, Kivimaki J, Ostermann H. The Macintosh lateral substitution reconstruction for anterior cruciate deficiency. Int Orthop. 1993;17:224-7.

150. Dandy DJ, Gray AJR. Anterior cruciate ligament reconstruction with the Leeds-Keio prosthesis plus extra-articular tenodesis. Results after six years. J Bone Joint Surg (Br). 1994;76-B:193-7.

151. Amis AA, Scammell BE. Biomechanics of intra-articular and extra-articular reconstruction of the anterior cruciate ligament. J Bone Joint Surg (Br). 1993;75-B:812-7.

152. Jensen JE, Slocum DB, Larson RL, James SL, Singer KM. Reconstruction procedures for anterior cruciate lig- 


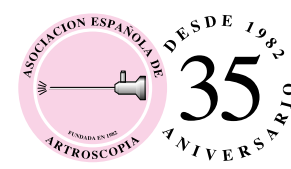

ament insufficiency: a computer analysis of clinical results. Am J Sports Med. 1983;11:240-8.

153. Strum GM, Fox JM, Ferkel RD, Dorey FH, Del Pizzo W, Friedman MJ, et al. Intraarticular vs intraarticular and extraarticular reconstruction for chronic anterior cruciate ligament instability Clin Orthop Rel Res. 1989;245:188-98.

154. Roth JH, Kennedy JC, Lockstadt H, McCallum CL, Cunning LA. Intraarticular reconstruction of the anterior cruciate ligament with and without extraarticular supplementation by transfer of the biceps femoris tendon. J Bone Joint Surg (Am). 1987;69-A:275-8.

155. Alm A, Liljedahl SO, Strömberg B. Clinical and experimental experience in reconstruction of the anterior cruciate ligament. Orthop Clin North Am. 1976;7:181-9.

156. Desplas B. Rupture du ligament croisé antéro-externe du genou gauche, réparation chirurgicale (operation de Hey-Groves). Bull Mem Soc Nat Chir. 1928;54:154-9.

157. Robineau M. Luxation du genou en avant. Bull Mem Soc Nat Chir. 1929;55:637-8.

158. Rosenberg TD, Rasmussen GL. The function of the anterior cruciate ligament during anterior drawer and Lachman's testing. Am J Sports Med. 1984;12:318-22.

159. Watanabe M. [Present status and future of arthroscopy]. Geka Chiryo. 1972;26:73-7.

160. Ikeuchi H. Arthroscopic treatment of the discoid lateral meniscus. Technique and long-term results. Clin Orthop Relat Res. 1982;167:19-28.

161. Ikeuchi $H$. The early days of arthroscopic surgery in Japan. Arthroscopy. 1988;4:222-5.

162. DeMaio M. Giants of orthopaedic surgery: Masaki Watanabe MD. Clin Orthop Relat Res. 2013;471:2443-8.

163. Pässler HH. [History of implant-free anterior cruciate ligament reconstruction]. Unfallchirurg. 2010;113:524-31.

164. Marić D, Garces G, Martinez A, Petković L. History of arthroscopy. Med Pregl. 2002;55:51-6.

165. Noyes FR, Mangine RE, Barber S. Early knee motion after open and arthroscopic anterior cruciate ligament reconstruction. Am J Sports Med. 1987;15:149-60.

166. Ludloff K. Der operative Ersatz des vorderen Kreuzbandes am Knie. Zentralbl Chir. 1927:54:3162-6.

167. Viernstein K, Keyl W. Operationen am Kniegelenk. En: Breitner B (ed.). Chirurgische Operationslehre, T. IV/2. Munich: Urban\&Schwarzenberg; 1973.

168. Mott W. Semitendinosus anatomic reconstruction for anterior cruciate ligament insufficiency. Clin Ortop Rel Res. 1983;172:90-2.

169. Meystre JL. Use of semitendinosus tendon for anterior cruciate ligament reconstruction. En: Jakob RP, Stäubli $\mathrm{HJ}$ (eds.). The knee and the cruciate ligaments. Berlín: Springer; 1992. pp. 376-83.
170. Gertel TH, Lew WD, Lewis JL, Stewart NJ, Hunter RE. Effect of anterior cruciate ligament graft tensioning direction, magnitude, and flexion angle on knee biomechanics. Am J Sports Med. 1993;21:572-81.

171. Fuss FK. Optimal replacement of the cruciate ligaments from the functional anatomical point of view. Acta Anat. 1991;140:260-8.

172. Hefzy MS, Grood ES, Noyes FR. Factors affecting the region of most isometric femoral attachments. Part II: the anterior cruciate ligament. Am J Sports Med. 1989;17:208-16.

173. Hefzy MS, Grood ES. Sensitivity of insertion locations on length patterns of anterior cruciate ligament fibers. J Biomech Eng. 1986;108:73-82

174. Penner DA, Daniel DM, Wood P, Mishra D. An in vitro study of anterior cruciate graft placement and isometry. Am J Sports Med. 1988;16:238-43.

175. Henning CE, Lynch MA, Glick KR. An in vivo strain gage study of the anterior cruciate ligament. Am J Sports Med. 1985;13:22-6.

176. Sidles JA, Larson RV, Garbini JL, Downey DJ, Matsen FA. Ligament length relationships in the moving knee. J Orthop Res. 1988;6:593-610.

177. Howe JG, Johnson RJ, Kaplan MJ, Fleming B, Jarvinen M. Anterior cruciate ligament reconstruction using quadriceps patellar tendon graft. Part I. Long-term followup. Am J Sports Med. 1991;19:447-57.

178. Shajrawi J, Aunallah J, Soudry M, Solomon H, Mendes DG, Boss JH. Quantification of the tissue response to a polyethylene prosthesis of the ACL in the goat: a histomorphometric study. Orthopaedics (IntEd). 1993;1:455-60.

179. Shino K, Nakata K, Horibe S, Inoue M, Nakagawa S. Quantitative evaluation after arthroscopic anterior cruciate ligament reconstruction. Allograft vs autograft. Am J Sports Med. 1993;21:609-16.

180. Cowan DJ. Reconstruction of the anterior cruciate ligament by the method of Kenneth Jones. Proc R Soc Med. 1963;58:336-8.

181. Odensten M, Lysholm J, Gillquist J. Long-term follow-up study of a distal iliotibial band transfer (DIT) for anterolateral knee instability. Clin Orthop Rel Res. 1983:176:129-35.

182. Artmann M, Wirtth CJ. Untersuchung über den funktionsgerechten Verlauf der vorderen Kreuzbandplastik. Z Orthop. 1974;112:160-5.

183. Yoshiya S, Andrish JT, Manley MT, Kurosaka M. Augmentation of anterior cruciate ligament reconstruction in dogs with prostheses of different stiffnesses. J Orthop Res. 1986;4:475-85.

184. Dorlot JM, Christel P, Meunier A, Sedel L, Witwoet J. Analyse du role mecanique des ligaments croisés 
dans la laxité antero-posterieure du genou. Int Orthop. 1983;7:91-7.

185. Schutzer SF, Christen S, Jakob RP. Further observations on the isometricity of the anterior cruciate ligament. Clin Orthop Rel Res. 1991;242:245-55.

186. Bradley J, Fitzpatrick D, Daniel D, Shercliff T, O'Connor J. Orientation of the cruciate ligament in the sagittal plane. J Bone Joint Surg (Br). 1988;70-B:94-9.

187. Segal P, Jacob M. The knee. Londres: Wolfe Medical Publ Ltd.; 1983.

188. Hertel P. Verletzung und Spannung von Kniebander. Unfallheilk. 1980;142:1-91.

189. Last RJ. Some anatomical details of the knee joint. J Bone Joint Surg (Br). 1948;30-B:683-8.

190. Jones R, Smith SA. On rupture of the crucial ligaments of the knee and on fractures of the spine of the tibia. Br J Surg. 1914;:1:70-89

191. Horwitz MT. An investigation of the surgical anatomy of the ligaments of the knee joint. Surg Gynecol Obstet. 1938;67:287-92.

192. Lembo R, Girgis FG, Marshall JL, Bartel DL. The antero-medial band (AMB) of the anterior cruciate ligament $(\mathrm{ACL})$ a linear and mathematical analysis. Anat Rec. 1975;181:409-11.

193. Dawkins GPC, Amis AA. A functional study of the structure of the anterior cruciate ligament, related to knee stability, injury mechanisms and prosthetic ligament reconstructions. J Bone Joint Surg (Br). 1985;67-B:844-47.

194. Walker PS, Rovick JS, Robertson DD. The effects of knee brace hinge design and placement on joint mechanics. J Biomech. 1988;21(11):965-74.

195. Friederich NF, O'Brien WR. Functional anatomy of the cruciate ligaments. En: Jakob RP, Stäubli HJ (eds.). The knee and the cruciate ligaments. Berlín: Springer, 1992. pp. 79-91.

196. Hewson GF. Drill guides for improving accuracy and in anterior cruciate ligament repair and reconstruction. Clin Orthop Rel Res. 1983;172:119-24.

197. Daniel DM, Malcolm LL, Losse G, Stone ML, Sachs R, Burks R. Instrumented measurement of anterior laxity of the knee. J Bone Joint Surg (Am). 1985;67-A:720-6.

198. Blazina MES, Kennedy JC. Surgical technique for prosthetic cruciate ligament replacement. Memphis: Richards Man Co Inc.; 1975.

199. Jimeno E, Ginebreda I, Ribas M, Vilarrubias JM. Tratamiento con plastias artificiales de los ligamentos cruzados de la rodilla en los deportistas. Rev Ortop Traumatol 1985;291B:351-63.

200. Cabot JR. Traitement chirurgical des lesions recentes de l'appareil capsuloligamentaire du genou dans le sport. Medicina Sportiva. 1957;12:620-36.
201. Cabaud HE, Feagin JA, Rodkey WG. Acute anterior cruciate ligament injury and augmented repair: experimental studies. Am J Sports Med. 1980;8:395-401.

202. Gillquist J, Liljedahl SO, Lindvall H. Reconstruction for old rupture of the anterior cruciate ligament. A follow-up study. Injury. 1971;2:271-8.

203. Kennedy J. Application of prosthetics to anterior cruciate ligament reconstruction and repair. Clin Orthop Rel Res. 1983;172:125-8.

204. Fujikawa K, Iseki F, Seddhom BB. Arthroscopy after anterior cruciate ligament reconstruction with Leeds-Keio ligament. J Bone Joint Surg (Br). 1989;71B:566-70.

205. Fujikawa K, Seedhom BB, Matsumoto H. Anterior cruciate reconstruction with the Leeds-Keio artificial ligament. Orthopaedics (Int Ed). 1995;3:53-64.

206. Mockwitz J, Contzen H. Alloplastic correction of chronic knee ligament instability with polyaethyleneterephtalate. Akt Probl Chir Orthop. 1983;26:110-5.

207. Bolton CW, Bruchman WC. The GORE-TEX expanded poly-tetra-fluoro-ethylene prosthetic ligament: an in vitro and in vivo evaluation. Clin Orthop Rel Res. 1985;196:202-13.

208. Paavolainen P, Makisalo S, Skutnabb K, Holmstrom T. Biologic anchorage of cruciate ligament prosthesis. Bone ingrowth and fixation of Gore-Tex ligament in sheep. Acta Orthop Scand. 1993;64:323-8.

209. McPherson GK, Mendenhall HV, Gibbons DF, Plenk H, Rottmann W, Sandford JB, et al. Experimental mechanical and histologic evaluation of the Kennedy ligament augmentation device. Clin Orthop Rel Res. 1985, 196:186-95.

210. Bejui J, Drouin G. Carbon fiber ligaments. CRC Critical Reviews in Biocompatibility. 1988;4:79-108.

211. Jenkins DHR. The repair of cruciate ligaments with flexible carbon fibre. A longer-term study of the induction of new ligaments and of the fate of the implanted carbon. J Bone Joint Surg (Br). 1978;60-B:520-2.

212. Grood ES, Noyes FR. Cruciate ligament prosthesis: strength, creep, and fatigue properties. J Bone Joint Surg (Am). 1976;58-A:1083-8.

213. Noyes FR, Butler DL, Grood ES, Zernicke RF, Hefzy MS. Biomechanical analysis of human ligament grafts used in knee-ligament repairs and reconstructions. I Bone Joint Surg (Am). 1984;66-A:344-52.

214. Lambert KL. Vascularized patellar tendon graft with rigid internal fixation for anterior cruciate ligament insufficiency. Clin Orthop Rel Res. 1983;172:85-9.

215. Dunn MG, Tria AJ, Kato YP, Bechler JR, Ochner RS, Zawadsky JP, et al. Anterior cruciate ligament reconstruction using a composite collagenous prosthesis. 


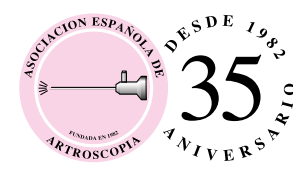

A biomechanical and histologic study in rabbits. Am J Sports Med. 1992;20:507-15.

216. Amiel D, Frank C, Harwood F, Fronek J, Akeson W. Tendons and ligaments: a morphological and biochemical comparison. J Orthop Res. 1984;1:257-265

217. Greis PE, Johnson DL, Fu FH. [Reconstruction of the cruciate ligaments with allogeneic transplants. Techniques, results and perspectives]. Orthopäde. 1993;22:386-91.

218. Horibe S, Shino K, Taga I, Inoue M, Ono K. Reconstruction of lateral ligaments of the ankle with allogeneic tendon grafts. J Bone Joint Surg (Br). 1991;73-B:802-5.

219. Noyes FR, Barber SD. The effect of an extra-articular procedure on allograft reconstructions for chronic ruptures of the anterior cruciate ligament. J Bone Joint Surg (Am). 1991;73-A:882-92.

220. Noyes FR, Barber S, Mangine RE. Bone-patellar ligament-bone and fascia lata allografts for reconstruction of the anterior cruciate ligament. J Bone Joint Surg (Am). 1990;72-A:1125-36.

221. Shino K, Inoue M, Horibe S, Hamada M, Ono K. Reconstruction of the anterior cruciate ligament using allogeneic tendon: long term follow-up. Am J Sports Med. 1990;18:457-65.

222. Shino K, Inoue M, Horibe S, Nagano J, Ono K. Maturation of allograft tendons transplanted into the knee: an arthroscopic and histological study. J Bone Joint Surg (Br). 1988;70-B:556-60.

223. Amiel D, Kleiner JB, Roux RD, Harwood FL, Akeson W. The phenomenon of "ligamentization": anterior cruciate ligament reconstruction with autogenous patellar tendon. J Orthop Res. 1986;4:162-172

224. Maeda A, Inoue M, Shino K, Nakata K, Nakamura $H$, Tanaka $M$, et al. Effects of solvent preservation with or without gamma irradiation on the material properties of canine tendon allografts. J Orthop Res. 1993;11:181-9.

225. Matawa MJ, Hutton WC. A biomechanical comparison between the central one-third patellar tendon and the residual tendon. Br J Sports Med. 1995;29:178-84.

226. Olson EJ, Harner CD, Fu FH, Silbey MB. Allograft bone and soft tissues. Clinical use of fresh, frozen soft tissue allograft. Orthopedics. 1992;15:1225-32.

227. Vaquero J, Calvo JA, Forriol F. Reconstrucción del ligamento cruzado anterior. Trauma (Mapfre). 2008;19(suppl 1): 22-38.

228. Arnoczky SP, Warren RF, Ashlock MA. Replacement of the anterior cruciate ligament using a patellar tendon allograft. An experimental study. J Bone Joint Surg (Am). 1986;66-A:376-85.

229. Cordrey LJ. A comparative study of fresh autogenous and preserved homogenous tendon grafts in rabbits. J Bone Joint Surg (Br). 1963;58-B:182-95.
230. Curtis RJ, De Lee JC, Drez DJ. Reconstruction of the anterior cruciate ligament with freeze dried fascia lata allografts in dogs. A preliminary report. Am J Sports Med. 1985;13:408-14.

231. Czitrom AA, Langer F, McKee N, Gross AE. Bone and cartilage allotransplantation. A review of 14 years of research and clinical studies. Clin Orthop Rel Res. 1986;208:141-5.

232. Peacock EE, Madden JW. Human composite flexor tendon allografts. Am Surg. 1967;166:62-92.

233. Friedlander GE. Current concepts review: bone banking. J Bone Joint Surg (Am). 1982;64-A:307-11.

234. Jackson DW, Grood ES, Wilcox P, Butler DL, Simon TM, Holden JP. The effects of processing techniques on the mechanical properties of bone-anterior cruciate ligament-bone allografts: an experimental study in goats. Am J Sports Med. 1988;16:101-5.

235. Jackson DW, Grood ES, Arnoczky SP, Butler DL, Simon TM. Cruciate reconstruction using freeze dried anterior cruciate ligament allograft and a ligament augmentation device (LAD): an experimental study in a goat model. Am J Sports Med. 1987;15:528-38.

236. Co FH, Skinner HB, Cannon WD. Effect of reconstruction of the anterior cruciate ligament on propioception of the knee and the heel strike transient. J Orthop Res. 1993;1:696-704.

237. Bircher E. Die Binnenverletzungen des Kniegelenkes. Basel: Benno Schwabe; 1929.

238. Bircher E. Über Kreuzbanverletzungen. Zentralbl Chir. 1930;57:2207.

239. Micheli E. Riconstruzione dei ligament crociati del ginocchio con tendine di canguro. Risolto a distanza. Boll Soc Piemont Chir. 1933;3:874-83.

240. Levitt RL, Malinin T, Posada A, Michalow A. Reconstruction of anterior cruciate ligaments with bone-patellar tendon-bone and achilles tendon allografts. Clin Orthop Relat Res. 1994;303:67-78.

241. DeFrére J, Franckart A. Freeze-dried fascia-lata allografts in the reconstruction of anterior cruciate ligament defects. A two to seven year follow-up. Clin Orthop Rel Res. 1994;303:587-96.

242. Valentí JR, Sala D, Schweitzer D. Anterior cruciate ligament reconstruction with fresh-frozen patellar tendon allografts. Int Orthop. 1994;18:210-4.

243. Paterson FWN, Trickey EL. Anterior cruciate ligament reconstruction using part of the patellar tendon as a free graft. J Bone Joint Surg (Br). 1986;76B:453-7.

244. Lysholm J, Gillquist J. Evaluation of knee ligament surgery results with special emphasis on use of a scoring scale. Am J Sports Med. 1982;10:150-4. 
245. Tegner Y, Lysholm J. Rating system in the evaluation of knee injuries. Clin Orthop Rel Res. 1985;198:43-9.

246. Burks RT, Haut RD, Lancaster BS. Biomechanical and histological observations on the dog patellar tendon after removal of its central one-third. Am J Sports Med. 1990;18:146-53.

247. Mastrokalos DS, Springer J, Siebold R, Paessler HH. Donor site morbidity and return to the preinjury activity level after anterior cruciate ligament reconstruction using ipsilateral and contralateral patellar tendon autograft. A retrospective, nonrandomized study. Am J Sports Med. 2005;33:85-93.

248. Cosgarea AJ, Sebastianelli WJ, DeHaven KE. Prevention of athrofibrosis after anterior cruciate ligament reconstruction using the central third patellar tendon autograft. Am J Sports Med. 1995;23:87-92

249. Fischer SE, Shelboume KD. Arthroscopic treatment of symptomatic extension block complicating anterior cruciate ligament reconstruction. Am J Sports Med. 1993;21:558-64.

250. Ejerhed L, Kartus J, Sernert N, Kohler K, Karlsson J. Patellar tendon or semitendinous tendon autografts for anterior cruciate ligament reconstruction? A prospective randomized study with a two-year follow-up. Am J Sports Med. 2003;31:19-25.
251. Marx RB, Jones EC, Angel M, Wickiewicz TL, Warren RF. Beliefs and attitudes of members of the American Academy of Orthopedic Surgeons regarding the treatment of anterior cruciate ligament injury. Arthroscopy. 2003;19:762-70.

252. Asociación Española de Artroscopia (AEA). Informe sobre el perfil de la cirugía artroscópica en España. Cuadernos de Artroscopia. 2001;8:10-21.

253. Serpell BG, Scarvell JM, Ball NB, Smith PN. Mechanisms and risk factors for non-contact $A C L$ injury in age mature arhletes who engage in field or court sports: a summary of literature since 1980. J Strength Cond Res. 2012;26:3160-76.

254. Campbell JD. The evolution and current treatment trends with anterior cruciate, posterior cruciate, and medial collateral ligament injuries. Am J Knee Surg. 1998;11:128-35.

255. Bach BR, Adalen KJ, Dennis MG, Carreira DS, Bojchuk J, Heyden JK, Bush-Joseph CA. Primary anterior cruciate ligament reconstruction using fresh-frozen, nonirradiated patellar tendon allograft. Am J Sports Med. 2005;33:284-92.

256. Bosch U, Kasperczyk J. Healing of the patellar tendon autograft after posterior cruciate ligament reconstruction-a process of ligamentization. Am J Sports Med. 1992;20:558-66. 